TITLE:

\title{
Enhanced predictions of wave impact pressure by improved incompressible SPH methods
}

$\operatorname{AUTHOR}(\mathrm{S})$ :

Khayyer, Abbas; Gotoh, Hitoshi; Shao, Songdong

\section{CITATION:}

Khayyer, Abbas .... [et al]. Enhanced predictions of wave impact pressure by improved incompressible SPH methods. Applied Ocean Research 2009, 31(2): 111-131

\section{ISSUE DATE:}

2009-04

URL:

http://hdl.handle.net/2433/87299

\section{RIGHT:}

c 2009 Elsevier Ltd. All rights reserved.; This is not the published version. Please cite only the published version.; この論文は出版社版でありませ ん。引用の際には出版社版をご確認ご利用ください。 


\title{
Enhanced predictions of wave impact pressure by improved Incompressible SPH methods
}

\author{
Abbas Khayyer $^{\mathrm{a}}$, Hitoshi Gotoh ${ }^{\mathrm{a}^{*}}$, Songdong Shao ${ }^{\mathrm{b}}$ \\ a Department of Urban and Environmental Engineering, Kyoto University, Katsura Campus, \\ Nishikyo-ku, Kyoto 615-8540, Japan \\ ${ }^{\mathrm{b}}$ School of Engineering, Design and Technology, University of Bradford, West Yorkshire, \\ BD7 1DP, United Kingdom
}

\begin{abstract}
A new criterion is proposed for a more efficient assessment of free-surface particles in a particle-based simulation. Enhanced wave impact simulations are carried out by improved Incompressible SPH (ISPH) methods. The first improvement is the same as that in the Corrected ISPH (CISPH; Khayyer et al., 2008) method and is proposed for the improvement of momentum conservation. The second improvement is achieved by deriving and employing a higher order source term based on a more accurate differentiation to obtain a less fluctuating and more accurate pressure field. The enhanced performance of improved ISPH methods is demonstrated through the simulation of several fluid impact simulations in comparison with the experimental data and simulation results by other numerical methods.
\end{abstract}

Keywords: SPH method, improved Incompressible SPH methods, particle method, wave impact pressure

\section{Introduction}

Past failures of coastal structures such as vertical breakwaters (Oumeraci, 1994) suggest that some of the conventional design methods based on solely static and quasi/static analysis are not entirely reliable. In addition, both theoretical (e.g. Cooker and Peregrine, 1992; Peregrine, 2003) and experimental studies (e.g. Hattori et al., 1994; Oumeraci et al, 2001; Bullock et al., 2007), indicate the necessity of dynamic analysis including wave impact loadings in design of the coastal structures. Therefore, development of reliable design tools which can appropriately predict the wave impact loadings or the so-called wave impact pressure becomes essential.

The numerical models developed for prediction of wave impact pressure are mainly based on

\footnotetext{
* Corresponding Author TEL: +81-75-383-3310 FAX: +81-75-383-3311

Email: gotoh@mbox.kudpc.kyoto-u.ac.jp
} 
Navier-Stokes equation which describes the motion of an incompressible, viscous fluid. Most Eulerian solvers of Navier-Stokes equation use grids and they should be coupled with a mathematical treatment of free surface. Christakis et al. (2002) and Kleefsman et al. (2005) employed modified versions of Volume Of Fluid (VOF) method (Hirt and Nichols, 1981) to treat the free surface in their wave impact calculations. Nevertheless, VOF-based models have the drawback of numerical diffusion arising from fixed-point interpolations of advection terms in both VOF function transport equation and Navier-Stokes momentum equation. A few sophisticated schemes such as the CIP method (Yabe et al., 2001) have been proposed to attenuate the numerical diffusion in an Eulerian grid-based calculation. Hu and Kashiwagi (2004) applied the CIP method in their grid-based wave impact calculations and obtained quite satisfactory results.

Recently, particle methods or the Lagrangian gridless methods have been applied in a wide variety of engineering applications including free-surface fluid flows. Due to their gridless feature, particle methods are inherently well-suited for the analysis of problems which include moving discontinuities or are characterized by large deformations. Moreover, because of their Lagrangian nature, such methods can analyze problems without numerical diffusion. Accordingly, particle methods provide a substantial potential for the simulation of free-surface fluid flows, especially those involving large deformations and fragmentations as in case of violent wave impacts on coastal structures.

The Smoothed Particle Hydrodynamics (SPH; Gingold and Monaghan, 1977; Lucy, 1977) is one of the earliest particle methods invented for modeling astrophysical phenomena. Since then, it has been extended to model a wide range of engineering applications including incompressible free-surface fluid flows by treating the flow as Weakly Compressible (WCSPH) with an appropriate equation of state (e.g. Monaghan, 1994), or strictly Incompressible (ISPH) through solving a Poisson Pressure Equation (e.g. Shao and Lo, 2003). The Moving Particle Semi-implicit (MPS; Koshizuka and Oka, 1996) is another particle method developed for simulation of incompressible fluid flows.

Despite their inherent capability for simulation of violent free-surface fluid flows, particle methods have a few drawbacks which may significantly affect their accuracy and performance. Such drawbacks are mainly caused by the interpolation nature of particle methods; that is, the local kernel-based interpolations on the basis of moving particles. Non-exact conservation of momentum (Bonet and Lok, 1999; Khayyer et al., 2008; Khayyer and Gotoh, 2008, 2009), lack of interpolation completeness (Liu et 
al, 1993) and existence of spurious pressure fluctuations (Gotoh et al., 2005) are among the major shortcomings associated with particle methods.

The accuracy and performance of particle methods have been enhanced by applying corrective techniques correcting either the kernel function itself or its gradient to improve the completeness of kernel interpolants and/or enhance the conservation of momentum, as in case of Corrected SPH (CSPH; Bonet and Lok, 1999) or Corrected Incompressible SPH (CISPH; Khayyer et al., 2008) methods. In a recent work, Khayyer and Gotoh (2008) improved the performance of MPS method by deriving an anti-symmetric pressure gradient term which guarantees the conservation of both linear and angular momentum.

To resolve the problem of spurious pressure fluctuation in their WCSPH calculation, Colagrossi and Landrini (2003) re-initialized the density field at distinctive time steps through applying a first-order accurate interpolation scheme via the employment of a moving-least-square kernel approximation. A more accurate interpolation scheme improves the consistency of mass-density-occupied area and accordingly results in a less fluctuating and more accurate source term for pressure equation (equation of state). Hence, a less fluctuating and more accurate pressure field would be obtained. In contrast to WCSPH method, the ISPH method employs a Poisson Pressure Equation (PPE) in which the pressure is a direct function of the time rate of change of density rather than the density itself. Accordingly, to obtain a less fluctuating and more accurate pressure field by the ISPH method, a more accurate source term of PPE based on a higher order calculation of the time rate of change of density should be derived.

In this paper, we propose a criterion for a more accurate and more efficient assessment of free-surface particles in particle-based simulations. Improved versions of ISPH method are applied for enhanced predictions of wave impact pressure. Improvements are achieved by improving the momentum conservation properties of ISPH formulations and by deriving and employing a higher order source term for PPE

The paper is organized in the following way. A brief explanation of ISPH method is presented in second chapter. The third chapter is allocated to the introduction of two modifications applied in this study. The first modification is the application of a corrective function derived based on a variational approach to ensure the angular momentum conservation of ISPH formulations (CISPH; Khayyer et al., 2008). By revisiting the derivation of Poisson Pressure Equation (PPE) in ISPH method, a higher order 
source term is derived as the second modification. The CISPH method modified by the higher order source term will be given the name CISPH-HS (CISPH with a Higher order Source term). In the forth chapter, the improvement achieved by applying the higher order source term is shown by two simple tests. In the fifth chapter, the improved ISPH methods are applied to the simulation of dam break with impact problems with comparing the results to the experiment (Hu and Kashiwagi, 2004; Zhou et al. 1999), CIP-based results (Hu and Kashiwagi, 2004) and results by some other numerical models. In the second section of this chapter, a new criterion is proposed for a more accurate assessment of free-surface particles. Finally, in the sixth chapter the enhanced calculation of wave impact pressure by improved ISPH methods is demonstrated by simulating a flip-through impact (Hattori et al, 1994).

\section{Incompressible SPH Method}

In this chapter, the ISPH method is briefly explained. Detailed descriptions are provided by Shao and Lo (2003) or Gotoh et al. (2004). The primary feature of particle methods is the discretization of the problem by use of moving computational points (or particles). The domain is discretized into a set of discrete particles the motion of each is governed by the conservation laws of continuum dynamics, in case of fluids, the continuity and Navier-Stokes equations:

$\frac{1}{\rho} \frac{\mathrm{D} \rho}{\mathrm{D} t}+\nabla \cdot \boldsymbol{u}=0$

$\frac{\mathrm{D} \boldsymbol{u}}{\mathrm{D} t}=-\frac{1}{\rho} \nabla p+\boldsymbol{g}+v \nabla^{2} \boldsymbol{u}$

where $\boldsymbol{u}=$ particle velocity vector; $t=$ time; $\rho=$ fluid density; $p=$ particle pressure; $\boldsymbol{g}=$ gravitational acceleration vector and $v=$ laminar kinematic viscosity. The left hand side of Eq. 2 denotes the Lagrangian differentiation involving the advection term. In the particle methods, including the ISPH method, the advection term is automatically calculated through the tracking of particle motion; hence, the numerical diffusion arising from the successive interpolation of the advection function in Eulerian grid-based methods is controlled without the need for a sophisticated scheme. The basic ISPH formulations are summarized as follows.

The density at particle $i$ is calculated by summing over the contributions of the neighboring particles:

$\rho_{i}=\sum_{j} m_{j} W\left(\left|\boldsymbol{r}_{i}-\boldsymbol{r}_{j}\right|, h\right)=\sum_{j} m_{j} W_{i j}$ 
where the subscript $i$ and $j$ denote the physical quantity corresponding to target particle $i$ and its neighboring particle $j ; \boldsymbol{r}=$ particle position; $m=$ mass of particle; $W=$ an interpolation weighting function and $h=$ the smoothing length taken as 1.2 times of initial particle spacing in this study.

The pressure gradient term is expressed in an anti-symmetric form as:

$$
\left(\frac{1}{\rho} \nabla p\right)_{i}=\sum_{j} m_{j}\left(\frac{p_{i}}{\rho_{i}^{2}}+\frac{p_{j}}{\rho_{j}^{2}}\right) \nabla_{i} W_{i j}
$$

By anti-symmetric we mean that pressure gradient calculated between particles $i$ and $j$ (target particle $i$ and its neighboring particle $j$ ) is equal and opposite to that calculated between particles $j$ and $i$ (target particle $j$ and its neighboring particle $i$ ). The anti-symmetric expression of interparticle accelerations (and hence forces) has a strong physical meaning as it guarantees the Newton's third law of motion as well as momentum conservation in a particle-based simulation (Khayyer and Gotoh, 2008, 2009).

The strain-based viscosity term is formulated as (Khayyer et al., 2008):

$$
\left(v \nabla^{2} \boldsymbol{u}\right)_{i}=\left(\frac{1}{\rho} \nabla \cdot \boldsymbol{T}\right)_{i}=\sum_{j} m_{j} \frac{8 v_{0} \rho_{j}}{\left(\rho_{i}+\rho_{j}\right)^{2}} \boldsymbol{S}_{j: i} \cdot \nabla_{i} W_{i j}
$$

where $v_{0}=$ the laminar kinematic viscosity and $\boldsymbol{S}_{j: i}=$ strain rate tensor of a neighboring particle $j$ with respect to the target particle $i$. In two dimensions, the strain rate tensor can be written as:

$$
\boldsymbol{S}_{j: i}=\left[\begin{array}{lc}
\left(\frac{\partial u}{\partial x}\right)_{j: i} & \left(\frac{1}{2}\left[\frac{\partial u}{\partial y}+\frac{\partial v}{\partial x}\right]\right)_{j: i} \\
\left(\frac{1}{2}\left[\frac{\partial u}{\partial y}+\frac{\partial v}{\partial x}\right]\right)_{j: i} & \left(\frac{\partial v}{\partial y}\right)_{j: i}
\end{array}\right] ;\left(\frac{\partial u}{\partial x}\right)_{j: i}=\frac{u_{j i}}{r_{i j}} \frac{x_{j i}}{r_{i j}}=\frac{u_{j}-u_{i}}{r_{i j}} \frac{x_{j}-x_{i}}{r_{i j}}
$$

where $u$ and $v=$ components of particle velocity in $x$ and $y$ directions, respectively. The Laplacian for pressure is expressed as:

$\nabla \cdot\left(\frac{1}{\rho} \nabla p\right)_{i}=\sum_{j} m_{j} \frac{8}{\left(\rho_{i}+\rho_{j}\right)^{2}} \frac{p_{i j} \boldsymbol{r}_{i j} \cdot \nabla_{i} W_{i j}}{\left|r_{i j}\right|^{2}}$

The calculation process in ISPH method is similar to that in MPS method (Koshizuka and Oka, 1996); that is, an iterative prediction-correction process composed of two main steps. The first prediction step is an explicit calculation to obtain intermediate or temporal velocities under the given viscosity and gravity terms. In the temporal flow field calculated by the first process, the mass conservation is not satisfied; in other words, the densities $\rho^{*}$ that are calculated at the end of first process deviate from the constant $\rho_{0}$; thus, a second corrective process is required to adjust the particle densities to initial constant values prior 
to the time step. In the second process, the intermediate particle velocities are updated implicitly through solving a Poisson Pressure Equation (PPE) derived as (Shao and Lo, 2003):

$\nabla \cdot\left(\frac{1}{\rho_{k}^{*}} \nabla p_{k+1}\right)_{i}=\frac{\rho_{0}-\left(\rho_{k}^{*}\right)_{i}}{\rho_{0}(\Delta t)^{2}}$

where $\Delta t=$ calculation time step; and $k$ denotes the step of calculation. In the ISPH method, if the density calculated at particle $i$ satisfies the following condition:

$\rho_{i}<\beta \rho_{0}$

the particle is considered as a free-surface particle (constant $\beta=0.99$ ) for which the zero pressure boundary condition is applied (Shao and Lo, 2003).

\section{Improved ISPH Method}

\subsection{Improved ISPH; momentum conservation}

All computational models for simulating fluid flows are based on the fundamental principles of physics including mass and momentum conservation. Particle methods are not an exception; however, because of particle-based discretization, local (and thus global) conservation of momentum may not be guaranteed in a particle-based calculation. In the ISPH method, the pressure interacting forces are anti-symmetric in addition to being radial (acting on the same line as position vector $\boldsymbol{r}_{i j}$ ); thus, both linear and angular momentum are preserved in case of pressure interacting forces (Khayyer et al., 2008). On the other hand, the viscous interacting forces that are obtained from a tensor-type strain-based viscosity do not necessarily act along the position vector $\boldsymbol{r}_{i j}$; hence, conservation of angular momentum will not be ensured in an ISPH calculation with tensor-type strain-based viscosity term.

Conservation of angular momentum can be guaranteed by ensuring the invariance of potential energy with respect to rigid body motions as shown by Bonet and Lok (1999). Khayyer et al. (2008) have employed a corrective technique proposed by Bonet and Lok (1999) to correct the gradient of kernel function in calculation of viscous interacting forces. The proposed method has been given the name Corrected ISPH (CISPH; Khayyer et al., 2008). The first modification employed in this study is the same as the one employed in the development of CISPH method; that is, correction of kernel gradients by applying a corrective matrix $\boldsymbol{L}$ :

$\widetilde{\nabla}_{i} W_{i j}=\boldsymbol{L}_{i} \nabla_{i} W_{i j}$ 
where $\boldsymbol{L}$ is derived as (Bonet and Lok, 1999):

$\boldsymbol{L}_{i}=\left(\sum V_{j} \nabla_{i} W_{i j} \otimes\left(\boldsymbol{r}_{j}-\boldsymbol{r}_{i}\right)\right)^{-1}$

The above correction ensures the exact calculation of the gradient of any linear velocity field. Furthermore, it guarantees the conservation of angular momentum since the internal forces are derived from a variational principle. Seeing that the pressure gradient term in ISPH method preserves both linear and angular momentum, the above correction is applied only during the calculation of viscous accelerations.

\subsection{Improved ISPH; source term in Poisson Pressure Equation}

In this section, we revisit the derivation of Poisson Pressure Equation (PPE) in the ISPH method and derive another formulation for the source function. The PPE (Eq. 8) in ISPH method has been derived from the mass conservation law (Eq. 1) as follows (Khayyer and Gotoh, 2009):

$\frac{1}{\rho_{0}}\left(\frac{\mathrm{D} \rho}{\mathrm{D} t}\right)_{i}^{*}+\nabla \cdot\left(\Delta \boldsymbol{u}_{k}^{* *}\right)_{i}=0 \quad ; \quad \Delta \boldsymbol{u}_{k}^{* *}=\boldsymbol{u}_{k+1}-\boldsymbol{u}_{k}^{*}=-\frac{\Delta t}{\rho_{k}^{*}} \nabla p_{k+1}$

where the superscript ${ }^{*}$ denotes the calculated quantities at the prediction step. In the ISPH method, the assumption is that at each time step the incompressibility is perfectly satisfied, that is to say, the temporal densities are exactly adjusted to $\rho_{0}$. Accordingly, by assuming a linear time variation of density Eq. 12 is written as:

$\frac{1}{\rho_{0}}\left(\frac{\rho_{k}^{*}-\rho_{0}}{\Delta t}\right)_{i}+\nabla \cdot\left(\Delta \boldsymbol{u}_{k}^{* *}\right)_{i}=0$

From Eqs. 12 and 13, the PPE (Eq. 8) is obtained and solved in the ISPH method. However, in reality because of the errors generated from the particle-based discretization of governing equations and the solution process of the system of linear equations, the calculated density at each time step and at a typical target particle $i$ would not be exactly equal to $\rho_{0}$. As a result, calculation of time variation of $\rho$ (= $\mathrm{D} \rho / \mathrm{D} t$ ) would be contaminated by numerical errors resulting in spurious pressure fluctuations and thus, an inaccurate pressure field. Here, another approach is applied for a more accurate calculation of $\mathrm{D} \rho / \mathrm{D} t$ at a target particle $i$ and at the prediction step of a typical time step $t$. From Eq. 3, we can write:

$$
\left(\frac{\mathrm{D} \rho}{\mathrm{D} t}\right)_{i}^{*}=\left(\sum_{i \neq j} m_{j} \frac{\mathrm{D} W_{i j}}{\mathrm{D} t}\right)_{i}^{*}=\left(\sum_{i \neq j} m_{j}\left[\frac{\partial W_{i j}}{\partial r_{i j}} \frac{\partial r_{i j}}{\partial x_{i j}} \frac{\mathrm{d} x_{i j}}{\mathrm{~d} t}+\frac{\partial W_{i j}}{\partial r_{i j}} \frac{\partial r_{i j}}{\partial y_{i j}} \frac{\mathrm{d} y_{i j}}{\mathrm{~d} t}\right]\right)_{i}^{*}=\left(\sum_{i \neq j} m_{j} \nabla_{i} W_{i j} \cdot \boldsymbol{u}_{i j}\right)_{i}^{*}
$$


On the other hand, from Eq. 12:

$\nabla \cdot\left(\frac{\Delta t}{\rho_{k}^{*}} \nabla p_{k+1}\right)_{i}=\frac{1}{\rho_{0}}\left(\frac{\mathrm{D} \rho}{\mathrm{D} t}\right)_{i}^{*}$

Therefore from Eqs. 14 and 15, the modified PPE would be obtained as:

$\nabla \cdot\left(\frac{1}{\rho_{k}^{*}} \nabla p_{k+1}\right)_{i}=\frac{1}{\rho_{0} \Delta t}\left(\sum_{i \neq j} m_{j} \nabla_{i} W_{i j} \cdot \boldsymbol{u}_{i j}\right)_{i}^{*}$

The ISPH method modified by the above formulation is referred to as ISPH-HS (ISPH method with a Higher order Source term). It should be noted that the combination of Eq. 14 is exactly the same expression for the time rate of change of density by Monaghan (1992). Furthermore, by rewriting the numerator of the original source term of the PPE (Eq. 8) as a combination of density deviation corresponding to the correction step of a typical time step $t\left(\Delta \rho_{*}\right)$ and density error at time step $t+1$ $\left(\Delta \rho^{t+1}\right)$, Ataie-Ashtiani and Shobeyri (2008) proposed the same formulation as Eq. 16. This equation can also be derived by taking the divergence of both sides of second part of Eq. 12 and by applying the SPH formulation of velocity divergence proposed by Monaghan (1992), as shown by Lee et al. (2008).

One relevant question regarding to Eq. 16 might be that whether by applying this equation the incompressibility of fluid would be absolutely satisfied or not. In other words, whether or not the velocity field obtained after the correction step is perfectly projected onto a divergence free space. From Eq. 15, when the neighbouring particles approach a target particle (at the prediction step) and the fluid compresses, $\mathrm{D} \rho / \mathrm{D} t, \rho^{*} / \rho_{0}$ and accordingly the source term of the PPE would be increased. This would result in an increase in the interparticle pressure interacting forces that would repulse the approaching neighbouring particles (at the correction step). The increase in the source term of the PPE is obtained from a continuity equation and is proportional to the divergence of velocity deviation $\left(\Delta u^{* *}\right)$ or the volume change (amount of compressibility) at the prediction step. Theoretically and in the absence of numerical approximations the fluid incompressibility would be satisfied by applying the mentioned prediction-correction approach. However, satisfaction of fluid incompressibility does not only depend on the accuracy of PPE's source term, but in addition it is dependent upon the accuracy of numerical schemes employed for time integration as well as for discretization of differential operators (such as Laplacian and gradient).

Another advantage of the higher order source term (Eq. 16) with respect to the original source term 
(Eq. 8) is that calculation of PPE's source term by Eq. 16 would be less sensitive to the variation of calculation time step $(\Delta t)$ seeing that the denominator of the source term in Eq. 8 includes square of $\Delta t$. It should be noted here that in all the simulations performed in this paper the calculation time step has been chosen according to the Courant stability condition and a time resolution chosen as $0.1 d_{0}$ with $d_{0}$ being the particle size (Khayyer et al., 2008).

\section{Improved Pressure Calculation by Applying the Higher Order Source Term}

\subsection{Time variation of hydrostatic pressure}

In this section, the improvement in calculation of pressure by employment of the higher order source term is demonstrated by a simple test, that is, time variation of hydrostatic pressure at a fixed point (Khayyer and Gotoh, 2009). A schematic view of the computation domain for this test is shown by Fig. 1. The particle size is selected as $d_{0}=0.04 \mathrm{~m}$ and the fluid height is $h=1.04 \mathrm{~m}$. The calculated time series of pressure by ISPH and ISPH-HS methods are shown in Fig. 2(a). From Fig. 2(a), the ISPH-HS has resulted in a relatively less fluctuating and more accurate pressure calculation. The numerical errors in the pressure calculation by ISPH and ISPH-HS methods are approximately $2.25 \%$ and $1.50 \%$, respectively.

The time variation of calculated density at the measuring point $\mathrm{A}$ is shown in Fig. 2(b). From this figure, it is evident that the ISPH-HS method has provided a smoother time variation of density although the amplitude of variations is more than that in the results by ISPH method. Furthermore, in average the amount of compressibility by ISPH-HS appears to be less than that by the ISPH method for this specific test. The maximum amounts of compressibility by both the ISPH and ISPH-HS methods appear to be about $0.001 \%$.

\subsection{A designed sinusoidal pressure variation}

In order to further confirm the improved performance of the ISPH-HS method compared to the ISPH method, the same hydrostatic pressure calculation is carried out this time with a designed sinusoidal pressure variation (Khayyer and Gotoh, 2009). A spatially constant, time varying sinusoidal term is added to the initial source term of PPE at all particles in the domain as follows:

$S_{i}^{M}=S_{i}^{I}+A_{s t} \operatorname{Sin}\left(2 \pi t / T_{s t}+\pi\right)$

where $S_{i}^{M}$ and $S_{i}^{I}=$ the Modified and Initial source term of PPE at particle $i$, respectively; $t=$ calculation 
time; $A_{s t}$ and $T_{s t}=$ the amplitude and period of sinusoidal term variations, respectively. The amplitude of additional sinusoidal source term $\left(A_{s t}\right)$ has been chosen such that the amplitude of sinusoidal pressure oscillations would be about $2000 \mathrm{~N} / \mathrm{m}^{2}$. Moreover, $T_{s t}$ has been selected to be $0.02 \mathrm{~s}$.

Fig. 3(a-b) shows the time variation of pressure and density at measuring point A by both ISPH and ISPH-HS methods. The figure demonstrates an enhanced consistency of density and pressure variations in the calculation by ISPH-HS method. Even in the existence of relatively sharp variations of PPE's source term, the ISPH-HS method has not failed to provide a smooth and consistent variation of both density and pressure. On the other hand, Fig. 3(a) shows a clear degradation of the accuracy of ISPH method in the vicinity of peak and dip points. While such accuracy degradation cannot be observed in the time series of calculated density by ISPH method seen in Fig. 3(b).

\section{Simulation of Dam Break with Impact; Improved ISPH Methods VS. ISPH Method}

The flow generated after the break of a dam and its impact against a vertical wall has been widely used (e.g. Colagrossi and Landrini, 2003; Hu and Kashiwagi, 2004) as a test problem for the evaluation of numerical methods in reproducing a highly-deformed flow. In this chapter, the enhanced performance of improved ISPH methods is demonstrated by simulating two cases of dam break with impact problems. The first case corresponds to the experiment by Hu and Kashiwagi (2004). This case is simulated so that the results by improved ISPH methods can be compared to those by a CIP-based numerical model (Hu and Kashiwagi, 2004) in addition to being compared to the experimental data. The second dam break problem which corresponds to the experiment by Zhou et al. (1999) is simulated to further verify the enhanced accuracy of improved ISPH methods and to present a comparison of the numerical results with some other numerical data including those by a WCSPH method (Colagrossi and Landrini, 2003), a VOF method (Nielsen, 2003) and a (two-phase) hybrid VOF-Level Set method (Park et al., 2009).

\subsection{Dam break with impact (Hu and Kashiwagi, 2004)}

A dam break with impact is simulated by ISPH and improved ISPH (CISPH, ISPH-HS and CISPH-HS) methods. The classifications of the modifications are summarized in Table 1. In this table, CISPH-HS-ASA represents the CISPH-HS method with a new criterion for the Assessment of free-surface on the basis of nearly Symmetric Arrangement of non-free-surface particles. This new 
criterion will be described in details in section 5.2. The physical conditions and the particle size $\left(d_{0}=\right.$ $0.004 \mathrm{~m}$ ) are set equivalent and equal to those in the study by $\mathrm{Hu}$ and Kashiwagi (2004). A schematic view of the computational domain is shown in Fig. 4. Point A denotes the point where the pressure sensor is installed.

Fig. 5 depicts the snapshots of water particles together with the pressure field at $t=0.002 \mathrm{~s}$ just after the release of the dam. The pressure fields by ISPH and CISPH appear to be quite similar. This is also the case for the pressure field by ISPH-HS and CISPH-HS methods. From the figure, it appears that both ISPH and CISPH methods have overestimated the pressure as the maximum pressure $\left(\approx 2250 \mathrm{~N} / \mathrm{m}^{2}\right)$ is quite larger than the hydrostatic pressure $\left(\approx 1170 \mathrm{~N} / \mathrm{m}^{2}\right)$. On the other hand, the maximum pressure by both ISPH-HS and CISPH-HS appear to be close to the hydrostatic one.

The flow after the dam break is characterized by the development of a tongue of water spreading along the horizontal boundary. Later on, this tongue of water impacts upon the vertical wall producing a large impact pressure. Fig. 6 shows the calculated pressure fields by ISPH and improved ISPH methods at the impact instant, more precisely, the instant at which the peak impact pressure is recorded. Both ISPH and CISPH results are characterized by pressure noises resulting in indistinguishable pressure contours. Such noises in pressure field are smoothed in the snapshots by ISPH-HS and CISPH-HS. As a consequence, both ISPH-HS and CISPH-HS have provided improved pressure fields characterized by distinctive pressure contours.

After the impact, the water is deviated upwards and rises up the wall in form of a jet. In rising up the wall, the jet slows down under the restoring action of gravity and starts to reverse. Eventually, due to the oncoming flow, the jet overturns in form of a plunging wave and hits the underlying water. Fig. 7 illustrates such violent plunging jet impact at $t=0.75 \mathrm{~s}$. The ISPH method has not portrayed a clear image of plunging jet due to considerable unphysical particle dispersiveness. The CISPH method has resulted in an enhanced reproduction of the plunging jet, yet, the pressure field contains some irregularities particularly close to the toe of the wall and in the vicinity of the plunging jet impact. The pressure field by both ISPH-HS and CISPH-HS appears to be smoother and more regularly distributed, compared to ISPH and CISPH methods. Furthermore, enhanced preservation of momentum and application of a higher order source term has helped the CISPH-HS method to provide a clearer and more integrated plunging jet together with a smoother pressure field. Thus, at least from the qualitative aspects, the CISPH-HS method 
appears to provide the best results among the 4 methods.

Fig. 8 shows the time histories of calculated pressure at point A by ISPH and improved ISPH methods together with the experimental data by $\mathrm{Hu}$ and Kashiwagi (2004). In this figure, the experimental data represent the mean value of the measured pressure data in 8 repeated experiments by Hu and Kashiwagi (2004). The first pressure peak occurs at the impact instant $\left(t_{\mathrm{i} \text {-exp }}=\right.$ averaged experimental impact time $=$ $0.348 \mathrm{~s}$ ) with an averaged value of $p^{\mathrm{i}-\mathrm{exp}}=1576.90 \mathrm{~N} / \mathrm{m}^{2}$, while the second pressure peak is induced when the plunging jet hits the underlying water and initiates a jet splash-up $\left(t_{\text {sp-exp }}=\right.$ averaged experimental time of second pressure peak $=0.750 \mathrm{~s} ; p^{\text {sp-exp }}=1192.30 \mathrm{~N} / \mathrm{m}^{2}$ ). Both ISPH and CISPH methods have underestimated the impact instant $\left(t_{\mathrm{i}-\mathrm{SPH}}=0.320 \mathrm{~s} ; t_{\mathrm{i}-\mathrm{CISPH}}=0.330 \mathrm{~s}\right)$ and have considerably overestimated the first pressure peak or the impact pressure $\left(p^{\mathrm{i}-\mathrm{ISPH}}=3928.43 \mathrm{~N} / \mathrm{m}^{2} ; p^{\mathrm{i}-\mathrm{CISPH}}=4134.34 \mathrm{~N} / \mathrm{m}^{2}\right)$. Furthermore, both ISPH and CISPH methods have resulted in fluctuating pressure fields together with numerous false zero-pressure points. The ISPH-HS method has overestimated both the impact instant and the impact pressure $\left(t_{\mathrm{i}-\mathrm{ISPH}-\mathrm{HS}}=0.355 \mathrm{~s}, p^{\mathrm{i}-\mathrm{ISPH}-\mathrm{HS}}=2450.04 \mathrm{~N} / \mathrm{m}^{2}\right)$. The pressure by ISPH-HS is still fluctuating especially prior to the occurrence of second pressure peak. The best simulation-experiment agreement is achieved in case of CISPH-HS results. Furthermore, the CISPH-HS method has well predicted both the impact instant $\left(t_{\mathrm{i} \text {-CISPH-HS }}=0.345 \mathrm{~s}\right)$ and the second pressure peak instant $\left(t_{\mathrm{sp}-\mathrm{CISPH}-\mathrm{HS}}=\right.$ $0.752 \mathrm{~s}$ ). An acceptable estimation of both impact pressure and second pressure peak is also provided by CISPH-HS method $\left(p^{\text {i-CISPH-HS }}=1762.19 \mathrm{~N} / \mathrm{m}^{2} ; p^{\text {sp-CISPH-HS }}=1177.72 \mathrm{~N} / \mathrm{m}^{2}\right)$. Although an enhanced (less fluctuating and more accurate) pressure trace has been obtained by CISPH-HS method, still the results contain some false zero-pressure points. The existence of such false zero-pressure points is most likely because of the employment of a simple condition for the assessment of free-surface particles. In this paper we propose a simple criterion for assessment of free-surface particles. Detailed description of the proposed criterion is presented in next section.

Fig. 9(a) shows the time history of pressure at measuring point A by CISPH-HS and CISPH-HS-ASA methods. The false zero-pressure points seen in the results by CISPH-HS are efficiently removed by applying the new criterion in CISPH-HS-ASA method. Furthermore, the CISPH-HS-ASA method has resolved two distinctive pressure peaks. Fig. 9(b) shows a comparison between the results by CISPH-HS-ASA and those by the CIP calculation (Hu and Kashiwagi, 2004). The CIP method has well calculated the first impact pressure as well as its rise time. However, the second pressure peak is 
underestimated in both magnitude and time of occurrence. Compared to the CIP results, the results by CISPH-HS-ASA appear to be slightly fluctuating, yet, the CISPH-HS-ASA method has better predicted the instant and magnitude of second pressure peak. Note that the CIP method applies high-order and sophisticated grid-based approximations together with a two-phase formulation, while, the CISPH-HS-ASA method employs a relatively simpler and more efficient particle-based approximation with a single-phase formulation.

As further evidence of the effectiveness of the new criterion for free-surface assessment, the snapshots by CISPH-HS and CISPH-HS-ASA methods at $t=0.750 \mathrm{~s}$ are shown in Fig. 10. The presented figure portrays a spatial distribution of false zero-pressure points and their efficient removal by applying a simple criterion.

To further verify the efficiency of the new criterion for free-surface assessment and to investigate the effect of spatial resolution on the performance and the accuracy of CISPH-HS and CISPH-HS-ASA methods, the same dam break problem has been re-simulated by considering different sets of initial particle spacings. Four additional simulations by considering two coarser $\left(d_{0}=0.006 \mathrm{~m} ; d_{0}=0.005 \mathrm{~m}\right)$ and two finer $\left(d_{0}=0.003 \mathrm{~m} ; d_{0}=0.002 \mathrm{~m}\right)$ initial particle spacings have been carried out by CISPH-HS-ASA method. In addition, two extra simulations corresponding to a coarser particle size $\left(d_{0}=\right.$ $0.006 \mathrm{~m})$ and finer one $\left(d_{0}=0.002 \mathrm{~m}\right)$ have been performed by the CISPH-HS method.

Fig. 11 illustrates the snapshots of water particles together with pressure field at $t=0.750 \mathrm{~s}$ by CISPH-HS and CISPH-HS-ASA methods with different initial particle spacings. The figure portrays the enhanced reproductions of the reversing plunging jet by increasing the spatial resolution. In addition, the improvements in spatial resolution have resulted in refined and smoother (spatial) pressure distributions. More importantly, the figure indicates the efficiency of the proposed criterion for free-surface assessment. Regardless of the initial particle spacing, the new criterion has enabled the CISPH-HS-ASA method to efficiently eliminate the unphysical zero-pressure points at the wall boundaries. From Fig. 11(a, e), a few false zero-pressure points can be observed at the wall boundaries in the snapshots by the CISPH-HS method.

Fig. 12(a) shows time history of calculated pressure at measuring point A (Fig. 4) corresponding to the CISPH-HS-ASA simulations with different spatial resolutions. From this figure, relatively more accurate and less fluctuating pressure variations have been obtained by enhancement of spatial resolution. 
Although the instant of the first pressure peak has been fairly well predicted by the CISPH-HS-ASA method with all the selected spatial resolutions, underestimations in prediction of second pressure peak instant are clear when the calculation has been preformed by a relatively coarse particle size $\left(d_{0}=0.006\right.$ $\left.\mathrm{m} ; d_{0}=0.005 \mathrm{~m}\right)$. The figure further confirms the effectiveness of the new criterion for free-surface assessment as there is no false zero-pressure point in the time histories of pressure calculated with different spatial resolutions.

Fig. 12(b) depicts the time variation of pressure at point A corresponding to the CISPH-HS and CISPH-HS-ASA calculations with initial particle spacings of $d_{0}=0.006 \mathrm{~m}$ and $d_{0}=0.002 \mathrm{~m}$. Although the improvement of spatial resolution has provided a relatively more stabilized pressure field in case of the CISPH-HS method, this method has not been able to portray a distinctive second pressure peak even with a relatively fine spatial resolution $\left(d_{0}=0.002 \mathrm{~m}\right)$. This is most likely due to an insufficient criterion for assessment of free-surface particles in CISPH-HS method. Because of this simplified criterion the results by CISPH-HS include a number of false zero-pressure points. The pressure traces by CISPH-HS-ASA method are superior to their corresponding results by CISPH-HS method as they contain less unphysical fluctuations, do not include false zero-pressure points and show a better agreement with the experiment.

Table 2 shows the (averaged) CPU time per calculation time step for ISPH and improved ISPH calculations of the dam break simulations performed with an initial particle spacing of $d_{0}=0.004 \mathrm{~m}$. From this table the required CPU time by ISPH and improved ISPH methods are about the same. Furthermore, employment of corrective terms by CISPH and CISPH-HS has increased the CPU time by about 4.0\%. Application of a new criterion for free-surface assessment has resulted in a slight increase of about $0.5 \%$ in the CPU time required by the CISPH-HS-ASA method. From this table, application of a higher order source term has brought about a slight decrease of about $2.0 \%$ in the CPU time which is most probably due to a faster iterative pressure solution process. The numerical calculations of the present paper have been carried out by a single CPU Intel Core ${ }^{\mathrm{TM}} 2$ Duo $2.40 \mathrm{GHz}$ with $2.00 \mathrm{~GB}$ system memory.

Table 3 shows the (averaged) CPU time per calculation time step for CISPH-HS-ASA calculations performed with different spatial resolutions. From this table and Fig. 12(a), an important fact can be deduced, that is, the need for selection of an optimum particle size by considering the desirable accuracy and the computational efficiency. For example from Fig. 12(a), an increase of spatial resolution from $d_{0}=$ 
$0.004 \mathrm{~m}$ to $d_{0}=0.003 \mathrm{~m}$ or from $d_{0}=0.004 \mathrm{~m}$ to $d_{0}=0.002 \mathrm{~m}$ has not significantly improved the performance of CISPH-HS-ASA in predicting the instants and magnitudes of first and second pressure peaks. Nevertheless, from Table 3, the required CPU time for CISPH-HS-ASA calculation with $d_{0}=$ $0.004 \mathrm{~m}$ is about 2.2 and 6.0 times faster than the calculations performed with $d_{0}=0.003 \mathrm{~m}$ and $d_{0}=$ $0.002 \mathrm{~m}$.

\subsection{A simple criterion for assessment of free-surface particles}

In most particle-based calculations of incompressible free-surface fluid flows, the free-surface particles are assessed by a simple condition based on the fact that the calculated density (or particle number density as in case of the MPS method) drops sharply at the free-surface boundary. The free-surface condition has been expressed by Eq. 9 in calculations by the ISPH method (e.g. Shao and Lo, 2003). In calculations by the MPS method (e.g. Koshizuka and Oka, 1996), it is expressed as:

$n_{i}<\beta n_{0}$

where $n_{i}$ and $n_{0}$ correspond to the calculated particle number density at particle $i$ and initial particle number density, respectively. In Eqs. 9 and 18, constant $\beta$ is chosen as slightly smaller than 1.0 (e.g. $\beta=$ 0.97 in Koshizuka and Oka, 1996; Gotoh and Sakai, 2006; $\beta=0.99$ in Shao and Lo, 2003). By applying the above free-surface condition, the particles at the free-surface boundary would be evaluated as free-surface particles and the zero-pressure boundary condition would be applied to these particles. However, in addition to the particles at the free-surface boundary numerous other particles located inside the computational domain may also be assessed as free-surface particles. This fact is evident in the studies by Khayyer et al. (2008) or Khayyer and Gotoh (2008).

The incorrect assessment of inner particles as free-surface arises from the fact that in a particle-based simulation, the calculated density at one typical particle $i$ is a direct function of the instantaneous relative positions of its neighboring particles $j$ (Eq. 3). The motions of such neighboring particles are calculated by some particle-based approximations (Eqs. 4 and 5). Hence, the density approximation on the basis of instantaneous relative particle positions would contain some fluctuations. In case of a rapidly-varied flow such as a dam break flow, the density fluctuations would become more significant. Thus, assessment of free-surface particles solely by the simple mentioned condition (Eqs. 9 or 18) would not always be appropriate. Here, we propose a criterion for more efficient and accurate assessment of free-surface 
particles in a particle-based calculation of incompressible free-surface fluid flows.

The new criterion is simply based on the fact that for a free-surface particle we have a non-symmetric distribution of neighboring particles (Fig. 13(a)). For this reason, the summation of either $x$-coordinate or $y$-coordinate of relative particle positions in the neighborhood of target particle $i$ would be larger than at least the initial particle spacing or the diameter of one particle $\left(=d_{0}\right)$. On the other hand, a particle with almost symmetric configuration of neighboring particles (Fig. 13(b)) should not be regarded as a free-surface particle although the particle density at that particle might become smaller than the boundary value $\left(=\beta \rho_{0}\right)$. Thus, the criteria for free-surface assessment are expressed by Eqs. 9 and 19:

$$
\left|\sum_{i \neq j}^{M} x_{i j}\right|>\alpha \quad \text { or } \quad\left|\sum_{i \neq j}^{M} y_{i j}\right|>\alpha
$$

where $M=$ number of neighboring particles; $\alpha=d_{0}$. Conditions expressed by Eq. 19 can be checked for particles with densities in the range of $0.9 \rho_{0}$ and $\beta \rho_{0}$. The CISPH-HS method with the new criterion for free-surface assessment has been given the name CISPH-HS-ASA (as shown in Table 1).

\subsection{Dam break with impact (Zhou et al., 1999)}

In order to further examine the enhanced precision of improved ISPH methods and to compare the results with those by improved WCSPH (Colagrossi and Landrini, 2003), VOF (Nielsen, 2003), Fluent (Abdolmaleki et al., 2004) and VOF-Level Set (Park et al., 2009) calculations, another case of dam break with impact is simulated. Conditions of the simulation correspond to the experiment by Zhou et al. (1999). A schematic sketch of the calculation domain is shown in Fig. 14. Point B indicates the pressure measuring point. The particle size is selected as $d_{0}=0.012 \mathrm{~m}$.

Fig. 15(a-c) shows the snapshots by CISPH-HS-ASA illustrating (a) the development of the reversing jet, (b) the impact of the reversing jet and (c) development of the splash-up. From the qualitative aspects, the CISPH-HS-ASA method has provided a clear reproduction of the reversing jet, its impact and resultant splash-up together with a refined pressure field.

In order to demonstrate the step-by-step improvements, the snapshots by ISPH and improved ISPH methods are shown at a typical time instant in Fig. 16. The snapshot by ISPH method is characterized by significant unphysical particle dispersiveness together with an irregular pressure distribution. The CISPH method has provided a clearer image of the jet with less unphysical dispersive particle motions; however, 
the noise in pressure field is still present. The pressure calculation by ISPH-HS method is superior to that by ISPH or CISPH methods; yet, analogous to the ISPH method, the ISPH-HS method has the drawback of unphysical particle dispersiveness. The enhancement in both free-surface profile and pressure field is evident in the snapshot by CISPH-HS method. Nevertheless, there exist a few false zero-pressure points at the wall boundaries. Such false zero-pressure points are efficiently removed in the snapshot by CISPH-HS-ASA method.

Fig. 17 depicts the snapshots by ISPH and improved ISPH methods as well as the results by improved WCSPH (Colagrossi and Landrini, 2003) and Boundary Element Method (BEM; Greco et al., 2004). Compared to the snapshots by ISPH and CISPH methods, the results by the methods employing a higher order source term are in better agreement with those by improved WCSPH (Colagrossi and Landrini, 2003) and BEM (Greco et al., 2004) methods.

Fig. 18(a) shows the experimental and calculated time variation of pressure at measuring point B. Similar to previous calculations, the results by ISPH and CISPH methods contain fluctuations large in amplitude, high in frequency. Thus, these two methods have not been able to provide an acceptable sketch of pressure time history. On the other hand, both the ISPH-HS and CISPH-HS methods have portrayed a significantly enhanced pressure trace in a better agreement with the experiment. The agreement in case of CISPH-HS results appears to be better than that in case of ISPH-HS results. Nevertheless, the calculated pressure at the wall boundary by both ISPH-HS and CISPH-HS methods contains numerous false zero-pressure points. Such false zero-pressure points are again efficiently removed by the CISPH-HS-ASA method as shown in Fig. 18(b). In addition to that, the CISPH-HS-ASA method has resulted in a further improved pressure trace in a better agreement with the experiment.

In Fig. 18(c) the CISPH-HS and CISPH-HS-ASA results are shown together with several other numerical results by improved WCSPH (Colagrossi and Landrini, 2003), VOF (Nielsen, 2003) and two-phase VOF-Level Set (Park et al., 2009) methods as well as the Fluent (Abdolmaleki et al., 2004) software. The Fluent results (Abdolmaleki et al., 2004) depict two distinctive pressure peaks, however, with a considerable overestimation. The VOF results (Nielsen, 2003) show a clear but delayed and overestimated second pressure peak, while they do not reveal the first pressure peak. The best grid-based results correspond to the two-phase VOF-Level Set method (Park et al., 2009), although this method has overestimated the instants of occurrence and magnitudes of both first and second pressure peaks. The 
single-phase improved WCSPH (Colagrossi and Landrini, 2003) has given a fairly good estimation of the first pressure peak and its rise time; nevertheless, its magnitude $\left(p^{1 \mathrm{st} \text {, SWCSPH }} / \rho g H=0.553\right)$ is somewhat underestimated (first normalized pressure peak by experiment $=p^{\underline{1 s t} \text { exp }} / \rho g H=0.691$ ) while the instant of occurrence is slightly overestimated. Furthermore, the second pressure peak is delayed and overestimated. Followed by the second pressure peak and at $t(g / H)^{0.5}=8.32$, a sharp and relatively large pressure rise is seen in the results by single-phase improved WCSPH. Such unphysical pressure rise is almost removed by two-phase improved WCSPH (Colagrossi and Landrini, 2003). The pressure trace for the two-phase case is almost the same as that in the single-phase case; yet, the two-phase model has given the second pressure peak closer to the experimental one. After the occurrence of second pressure peak, the calculated pressure by two-phase improved WCSPH undergoes fluctuations mostly in the vicinity of the experimental data. The CISPH-HS method has provided an acceptable calculation of the first pressure peak $\left(p \frac{1 \text { st, CISPH-HS }}{\rho} \rho g H=0.714\right)$ and its rise time, although the occurrence is slightly delayed. The calculated second pressure peak by CISPH-HS is also delayed, however, its magnitude ( $p^{\text {2nd,CISPH-HS }} / \rho g H$ $=0.895)$ is quite close to the experimental one $\left(p^{2 \mathrm{nd} \text {, Exp }} / \rho g H=0.879\right)$. A sharp pressure rise similar to the one seen in the results by single-phase improved WCSPH, occurs at $t(g / H)^{0.5}=8.36$ in the calculation by CISPH-HS. Nonetheless, the magnitude of such pressure rise is much less than that in single-phase improved WCSPH results. Compared to the CISPH-HS, the CISPH-HS-ASA method has provided a less fluctuating and more accurate pressure calculation without the existence of false-zero pressure points.

Fig. 19(a-b) shows the time variation of water heights $h_{1}$ and $h_{2}$ at locations $(x / H)_{1}=0.825$ and $(x / H)_{2}$ $=1.653$ from the right vertical wall, respectively. Following Colagrossi and Landrini (2003), the height of the possibly present entrapped air is reduced from the total water level in view of the fact that the experiment (Zhou et al., 1999) has been carried out by use of standard capacitive wave gages being sensitive to the wetted portion of the wire. From Fig. 19(a) the numerical results by CISPH-HS-ASA, single-phase improved WCSPH and Fluent are in good agreement with the experiment before $t$ $(g / H)^{0.5} \approx 6.76$. Later, however, the experimental data appear to rise, while, the numerical data (except for two-phase VOF-Level Set; Park et al., 2009) tend to decrease. This decreasing tendency is more pronounced in the results by single-phase improved WCSPH method (Colagrossi and Landrini, 2003). The CISPH-HS-ASA results agree with the experiment reasonably well again from $t(g / H)^{0.5} \approx 10.0$ until $t$ $(g / H)^{0.5} \approx 13.0$. On the other hand, the water height results by improved WCSPH method, Fluent software 
and two-phase VOF-Level Set method are available only up to $t(g / H)^{0.5} \approx 8.5, t(g / H)^{0.5} \approx 9.0$ and $t$ $(g / H)^{0.5} \approx 10.0$, respectively. The two-phase VOF-Level Set method overestimates the instants and magnitudes of both first and second rises in water heights seen in Fig. 17(a). However, it provides a fairly good agreement with the experiment.

In case of $h_{2}$ (Fig. 19(b)), the numerical results (except for two-phase VOF-Level Set; Park et al., 2009) appear to overestimate the rise in water height at $t(g / H)^{0.5} \approx 7.10$. Later on, the results by single-phase improved WCSPH method show a sharp decreasing trend, resulting in underestimation of water height after $t(g / H)^{0.5} \approx 7.50$. The results by Fluent and CISPH-HS-ASA experience a milder decrease and overestimate the water height. The two-phase VOF-Level Set (Park et al., 2009) has overestimated the instant of the rise in water height, nevertheless it gives a better estimation of wave height variation from $t(g / H)^{0.5} \approx 7.00$ until $t(g / H)^{0.5} \approx 8.60$. From $t(g / H)^{0.5} \approx 9.00$ the wave height variation by CISPH-HS-ASA becomes quite consistent with the experiment until $t(g / H)^{0.5} \approx 12.0$, while the improved WCSPH, Fluent and two-phase VOF-Level Set results are not available at the later stage of the dam break.

\section{Simulation of a Flip-Through without Air Entrapment}

Wave impact pressure depends directly on the shape of the impacting wave. A gentle wave simply sloshes up and down, and reflects from the wall. A wave with a larger wave height may overturn and hit the wall. For the latter case an air pocket can be trapped, whereas, broken waves might already contain large quantities of air. At the margin, the wave front becomes nearly vertical, but it does not overturn. Instead, the wave through rapidly rises as an upward deflected jet at the wall. This phenomenon is called a "flip-through" (Cooker and Peregrine, 1992).

In this chapter the applicability of improved ISPH methods for prediction of wave impact pressure is investigated by simulating a flip-through without air entrapment corresponding to the experiment by Hattori et al. (1994). Simulation of a flip-through with air entrapment is postponed to our future works when we develop two-phase improved ISPH methods.

The physical and incident wave conditions are set equivalent to those in the experimental study by Hattori et al. (1994). A schematic sketch of the numerical domain is shown in Fig. 20(a). The characteristics of the incident wave are: incident wave height $H_{\mathrm{i}}=4.7 \mathrm{~cm}$; wave period $T=1.7 \mathrm{~s}$. 
Considering the prescribed physical conditions, the mentioned wave characteristics lead to a maximum wave height $\left(=H_{\mathrm{F}}\right)$ of $6.9 \mathrm{~cm}$ and a trough depth $\left(=h_{\mathrm{d}}\right)$ of $1.6 \mathrm{~cm}$ close to the wall. Hattori et al. (1994) measured the impact pressures by 4 pressure transducers (denoted by $\mathrm{P}_{1}-\mathrm{P}_{4}$ ) installed along the centerline of the wall as depicted in Fig. 20(b). The size of particles is chosen as $d_{0}=0.005 \mathrm{~m}$ for this calculation case.

Fig. 21(a-b) illustrates the ISPH and improved ISPH snapshots of water particles together with the pressure field during the simulated flip-through impact. The time of the snapshots is normalized following Hattori et al. (1994): $t^{*}=t C_{\mathrm{s}} / H_{\mathrm{F}} ; C_{\mathrm{s}}=1500 \mathrm{~m} / \mathrm{s}$; and $t^{*}=200$ refers to the time of maximum peak pressure recorded at $\mathrm{P}_{3}$. At $t^{*}=-800$, the pressure by ISPH-HS and CISPH-HS methods is characterized by distinctive pressure contours. On the other hand, the pressure by both ISPH and CISPH methods contains some noises. As a result, distinctive pressure contours are not evident in ISPH and CISPH snapshots. Moreover, the free-surface boundary in CISPH and CISPH-HS snapshots appear to be quite smoother than that in ISPH and ISPH-HS snapshots. The pressure disturbances in ISPH and CISPH snapshots are further increased at $t^{*}=0$. The existence of many zero-pressure particles that have been mistakenly assessed as free-surface particles is evident close to the vertical wall in both ISPH and CISPH snapshots. On the other hand, both ISPH-HS and CISPH-HS methods have resulted in relatively smoother pressure fields. Again the snapshot by CISPH appears to be superior in terms of both the free-surface profile and the pressure field.

At $t^{*}=200$ both ISPH-HS and CISPH-HS methods have provided relatively smooth pressure fields distinguished by distinctive pressure contours very similar to those computed by Cooker and Peregrine (1992). A schematic sketch of the pressure contours at the instant of maximum impact pressure in a flip-through computed by Cooker and Peregrine (1992) is shown in Fig. 22. The maximum impact pressure by both ISPH-HS and CISPH-HS methods is also predicted to be in the vicinity of the still water level which is in agreement with the computation by Cooker and Peregrine (1992). This fact has been pointed out by some other researchers (e.g. Kirkgoz, 1990; Hattori et al., 1994) and has been explained by the contraction of the horizontally and vertically moving water surfaces in the vicinity of the still water level (Hattori et al., 1994). The snapshots by ISPH and CISPH at $t^{*}=200$ are characterized by pressure disturbances particularly close to the vertical wall. As a consequence, both methods have not been able to portray an acceptable pressure field at the instant of maximum impact pressure in a flip-through impact. 
At $t^{*}=500$ both ISPH and CISPH methods have resulted in fluctuating pressure fields. Again the existence of pressure noise and false zero-pressure points is clear in the snapshots by ISPH and CISPH methods. The pressure fields by ISPH-HS and CISPH-HS are considerably more smoothly and regularly distributed. Analogous to the snapshots at other three previous instants, the main difference between ISPH-HS and CISPH-HS snapshots appears to be in the reproduction of the free-surface. In contrast to the ISPH method, the CISPH method has resulted in a smoother and mainly a one-particle-thick free-surface layer. This has also been the case in previous comparative computations by ISPH and CISPH (Khayyer et al., 2008).

Fig. 23 shows the experimental (Hattori et al., 1994) and calculated time variation of pressure at measuring points $\mathrm{P}_{1}-\mathrm{P}_{4}$. In this figure, $p^{*}\left(=p / \rho g H_{\mathrm{F}}\right)$ represents the normalized pressure. From the figure it is evident that neither ISPH nor CISPH could provide an acceptable pressure trace during a flip-through impact. Both methods have resulted in fluctuating pressure fields and lots of spurious zero-pressure points at all the measuring points $\mathrm{P}_{1}-\mathrm{P}_{4}$. On the contrary, the employment of a higher order source term in PPE by ISPH-HS and CISPH-HS methods has significantly enhanced the pressure calculation seeing that the pressure traces by these two methods are in better agreement with the experiment. At $\mathrm{P}_{1}$, both ISPH-HS and CISPH-HS methods have fairly well predicted the increase in pressure and its declination although both methods have slightly overestimated the pressure rise time as well as the pressure magnitude in the declination region. The calculated pressure by CISPH-HS method seems to be superior to that by

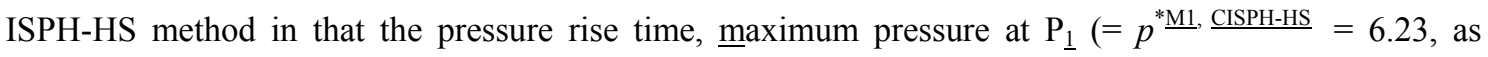
compared with the experimental value of $p^{* \mathrm{M} 1 \text {, Exp }}=5.59$ ) and the pressure declination are in better agreement with the experiment. The superiority of CISPH-HS to ISPH-HS becomes more revealed in the pressure plot at $\mathrm{P}_{2}$. The calculated pressure at $\mathrm{P}_{2}$ by CISPH-HS agrees relatively well with the experiment. The pressure rise time, its maximum value at $\mathrm{P}_{2}$ and the decrease in pressure after the rise are fairly well predicted by the CISPH-HS method. Although the pressure by ISPH-HS method is better than that by ISPH and CISPH methods, the pressure rise time and the magnitude of pressure between $t^{*}=300$ and $t^{*}$ $=600$ have been notably overestimated.

The ISPH-HS and CISPH-HS methods have also resulted in a significantly improved pressure trace at

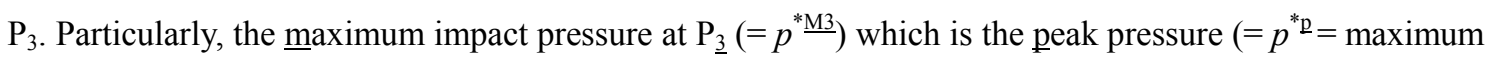
pressure on the vertical wall) is fairly well estimated by the ISPH-HS and CISPH-HS methods although 
some amount of overestimation exist $\left(p^{* \mathrm{M} 3, \underline{\mathrm{ISPH}-\mathrm{HS}}}=p^{* \mathrm{p}, \underline{\text { ISPH-HS }}}=11.26 ; p^{* \mathrm{M} 3, \underline{\mathrm{CISPH}-\mathrm{HS}}}=p^{* \mathrm{p}, \underline{\mathrm{CISPH}-\mathrm{HS}}}=\right.$ $\left.10.85 ; p^{* \mathrm{p}, \underline{\operatorname{Exp}}}=9.30\right)$. Both ISPH-HS and CISPH-HS methods have also well predicted the pressure declination at $\mathrm{P}_{3}$. The calculated pressure at $\mathrm{P}_{4}$ by ISPH-HS and CISPH-HS appears to be more fluctuating and less accurate than those calculated at $\mathrm{P}_{1}-\mathrm{P}_{3}$ by the same methods. This might be due to the incompleteness of interpolations (Liu et al., 1993) which becomes more dominant close to a boundary. Furthermore, because $\mathrm{P}_{4}$ is located close to the air-water interface, the two-phase flow effect would become important. Hence, two-phase improved ISPH methods are expected to provide better results.

In conclusion, seeing that both ISPH-HS and CISPH-HS methods have resulted in acceptable pressure traces at $\mathrm{P}_{1}-\mathrm{P}_{3}$ and more importantly, a satisfactory calculation of maximum pressure (which is equivalent to the peak pressure) at $\mathrm{P}_{3}$, these two methods appear to be applicable for an approximate calculation of non-aerated wave impact pressure on a coastal structure. For the prediction of aerated wave impact pressure; however, two-phase flow models should be developed.

It should be noted that for the case of the flip-through impact simulated in this section, the CISPH-HS method did not yield any spurious false zero-pressure point at least at the measuring points $\mathrm{P}_{1}-\mathrm{P}_{4}$. For this reason, we have not implemented a CISPH-HS-ASA calculation, although for a more violent case of flip-through or other violent wave impacts, the new criterion proposed in section 5.2 should be implemented.

\section{Concluding Remarks}

The paper presents enhanced predictions of wave impact pressure by improved Incompressible SPH (ISPH) methods. Enhanced calculation of wave impact pressure is achieved by applying two modifications. The first modification focuses on the momentum conservation properties of ISPH formulations. The Corrected ISPH (CISPH; Khayyer et al., 2008) method applies a corrective function derived on the basis of a variational approach (Bonet and Lok, 1999) to ensure the conservation of angular momentum in an ISPH calculation. As for the second modification, a higher order source term is derived after revisiting the derivation of original Poisson Pressure Equation (PPE) in ISPH method. It is shown that the higher order source term improves the consistency of density and pressure variations and results in a less fluctuating and more accurate pressure field. The ISPH and CISPH methods modified by the higher order source term have been given the names ISPH- $\underline{\mathrm{HS}}$ and CISPH- $\underline{\mathrm{HS}}$, respectively. 
The enhanced performance of improved ISPH methods in calculation of wave impact pressure is shown through the simulation of two cases of dam break with impact (Hu and Kashiwagi, 2004; Zhou et al., 1999) and a flip-through impact (Hattori et al., 1994). The simulation results are compared to the experimental data as well as the results by other numerical methods including improved Weakly Compressible SPH (WCSPH), CIP, VOF and VOF-Level Set methods, also the Fluent software. The comparisons clearly show the considerable improvements in calculation of wave impact pressure by ISPH-HS and CISPH-HS methods. The CISPH-HS method which benefits from two improvements tends to be superior to other improved ISPH methods as it provides both a significantly enhanced pressure field together with a more accurate reproduction of free-surface profile.

In addition to the mentioned improvements, a new criterion is proposed for a more efficient and accurate assessment of free-surface particles. The new criterion is proposed on the basis of nearly symmetric arrangement of non-free-surface particles. It has been demonstrated that application of the proposed criterion efficiently removes the false zero-pressure points that are mistakenly assessed as free-surface particles as a result of the employment of a simplified condition. In addition to its efficiency, the proposed criterion is simple and easy to be implemented.

The improved ISPH methods proposed in this paper are single-phase flow models. For the prediction of the aerated impacts with entrapped/entrained air, however, a two-phase improved ISPH method would be required (Gotoh and Sakai, 2006). Furthermore, as the proposed methods are 2D models with no description of Sub-Particle-Scale turbulence (Gotoh et al. 2001), the three-dimensionality of the flow and effect of small scale turbulent motions have not been taken into account. A 3D two-phase improved ISPH method with a SPS turbulence model should be developed to achieve a more realistic and more accurate calculation of wave impact pressure.

\section{References:}

Abdolmaleki, K., Thiagarajan, K. P. and Morris-Thomas, M. T., 2004. Simulation of the dam break problem and impact flows using a Navier-Stokes Solver, $15^{\text {th }}$ Australian Fluid Mechanics Conference, The University of Sydney, Sydney, Australia.

Ataie-Ashtiani B. and Shobeyri G., 2008. Numerical simulation of landslide impulsive waves by incompressible smoothed particle hydrodynamics, Int. J. Numer. Meth. Fluids, 56, 209-232. 
Bonet, J. and Lok, T. S., 1999. Variational and momentum preservation aspects of smooth particle hydrodynamic formulation. Comput. Meth. Appl. Mech. Eng. 180, 97-115.

Bullock, G.N., Obhrai, C., Peregrine, D.H. and Bredmose, H., 2007. Violent breaking wave impacts. Part 1: Results from large-scale regular wave tests on vertical and sloping walls. Coastal Engineering 54, $602-617$.

Colagrossi, A. and Landrini, M., 2003. Numerical simulation of interfacial flows by smoothed particle hydrodynamics. J. Comput. Phys., 191, 448-475.

Christakis, N, Allsop, N.W.H., Beale, R.G., Cooper, A.J. and Dennis, J.M., 2002. A Volume of Fluid numerical model for wave impacts at coastal structures. Water \& Maritime Engineering 154 (3), 159-168.

Cooker, M.J. and Peregrine, D.H., 1992. Wave impact pressures and its effect upon bodies lying on the sea bed. Coastal Engineering 18 (3-4), 205-229.

Gingold, R. A. and Monaghan, J. J., 1977. Smoothed particle hydrodynamics: theory and application to non-spherical stars. Mon. Not. R. Astron. Soc., 181, 375-89.

Gotoh, H., Ikari, H., Memita, T. and Sakai, T., 2005. Lagrangian particle method for simulation of wave overtopping on a vertical seawall. Coast. Eng. J. 47(2 \& 3), 157-181.

Gotoh, H. and Sakai, T., 2006. Key issues in the particle method for computation of wave breaking. Coastal Engineering 53(2-3), 171-179.

Gotoh, H., Shao, S. D. and Memita, T., 2004. SPH-LES model for numerical investigation of wave interaction with partially immersed breakwater. Coastal Eng. Jour., JSCE, 46(1), 39-63.

Gotoh, H., Shibahara, T. and Sakai, T., 2001. Sub-Particle-Scale turbulence model for the MPS method -Lagrangian flow model for hydraulic engineering. Comput. Fluid Dyn. J. 9(4), 339-347.

Greco, M., Landrini, M. and Faltinsen, O.M., 2004. Impact flows and loads on ship-deck structures, Journal of Fluids and Structures 19, 251-275.

Hattori, M., Arami, A. and Yui, T., 1994. Wave impact pressure on vertical walls under breaking waves of various types. Coastal Engineering 22 (1-2), 79-114.

Hirt, C. and Nichols, B. D., 1981. Volume of fluid (VOF) method for the dynamics of free boundaries, J. Comput. Phys. 39, 201-225.

Hu, C.H. and Kashiwagi, M., 2004. A CIP method for numerical simulations of violent free surface flows, 
Journal of Marine Science and Technology 9 (4), 143-157.

Khayyer, A., Gotoh, H. and Shao, S.D., 2008. Corrected Incompressible SPH method for accurate water-surface tracking in breaking waves. Coastal Engineering 55(3), 236-250.

Khayyer, A. and Gotoh, H., 2008. Development of CMPS method for accurate water-surface tracking in breaking waves. Coastal Engineering Journal, 50(2), 179-207.

Khayyer, A. and Gotoh, H., 2009. Modified Moving Particle Semi-implicit methods for the prediction of 2D wave impact pressure. Coastal Engineering, 56(4), 419-440.

Kirkgoz, M.S., 1990. An experimental investigation of a vertical wall response to breaking wave impact, Ocean Engineering 17(4), 379-391.

Kleefsman, K.M.T., Fekken, G., Veldman, A.E.P., Iwanowski, B. and Buchner, B., 2005. A Volume-of-Fluid based simulation method for wave impact problems. J. Comp. Phys. 206, 363-393.

Koshizuka, S. and Oka, Y., 1996. Moving particle semi-implicit method for fragmentation of incompressible fluid. Nuclear Science and Engineering 123, 421-434.

Lee, E.S., Moulinec, C., Xu, R., Violeau, D., Laurence, D. and Stansby, P., 2008. Comparisons of weakly compressible and truly incompressible algorithms for the SPH mesh free particle method, J. Comp. Phys. 227(18), 8417-8436.

Liu, W.K., Adee, J. and Jun, S., 1993. Reproducing kernel and wavelets particle methods for elastic and plastic problems. Advanced Computational Methods for Material Modeling, AMD 180/PVP 268 ASME, 175-190.

Lucy, L. B., 1977. A numerical approach to the testing of the fission hypothesis. Astron. J., 82, 1013-24.

Monaghan, J. J., 1992. Smoothed particle hydrodynamics. Ann. Rev. Astron. Astrophys. 30, 543-574.

Monaghan, J. J., 1994. Simulating free surface flows with SPH. J. Comput. Phys. 110, 399-406.

Nielsen, K. B., 2003. Numerical Prediction of Green Water Loads on Ships, Ph.D. thesis, Technical University Of Denmark, Lyngby, Denmark.

Oumeraci, H., 1994. Review and analysis of vertical breakwater failures - lessons learned. Coastal Engineering $22(1-2), 3-29$.

Oumeraci, H., Kortenhaus, A., Allsop, W., de Groot, H., Crouch, R., Vrijling, H. and Voortman, H., 2001. Probabilistic Design Tools for Vertical Breakwaters. A.A. Balkema, Lisse.

Park, I. R., Kim, K. S., Kim, J. And Van, S. H., 2009. A volume-of-fluid method for incompressible 
free-surface flows, Int. J. Numer. Meth. Fluids, DOI: 10.1002/fld.2000.

Peregrine, D.H., 2003. Water wave impact on walls, Ann. Rev. Fluid Mech. 35, 23-43.

Shao, S. D. and Lo, E. Y. M., 2003. Incompressible SPH method for simulating Newtonian and non-Newtonian flows with a free surface. Advances in Water Resources, 26 (7), 787-800.

Yabe, T., Xiao, F. and Utsumi, T., 2001. The Constrained Interpolation Profile method for multiphase analysis. J. Comp. Phys. 169, 556-593.

Zhou, Z. Q., Kat, J. O. D. and Buchner, B., 1999. A nonlinear 3-D approach to simulate green water dynamics on deck, Nantes, $7^{\text {th }}$ Intl. Conf. Num. Ship Hydrodynamics, Nantes. 
Fig. 1. A schematic sketch of the computational domain for hydrostatic pressure calculation

Fig. 2. Hydrostatic pressure calculation - time variation of (a) pressure and (b) density at measuring point $\mathrm{A}$

Fig. 3. Time variation of (a) pressure and (b) density at measuring point A in the hydrostatic pressure calculation plus a designed sinusoidal variation of source term of Poisson Pressure Equation

Fig. 4. A schematic sketch of the dam break simulation corresponding to the experiment by Hu and Kashiwagi (2004)

Fig. 5. Dam break simulation (Hu and Kashiwagi, 2004) - Snapshots of water particles at $t=0.002 \mathrm{~s}$

Fig. 6. Dam break simulation (Hu and Kashiwagi, 2004) - Snapshots of water particles at the impact instant when the maximum impact pressure is recorded

Fig. 7. Dam break simulation (Hu and Kashiwagi, 2004) - Snapshots of water particles at $t=0.75 \mathrm{~s}$

Fig. 8. Dam break simulation (Hu and Kashiwagi, 2004) - time histories of calculated pressure at point A by ISPH and improved ISPH methods

Fig. 9. Dam break simulation - Comparison between the experimental data (Hu and Kashiwagi, 2004),

(a) CISPH-HS and CISPH-HS-ASA, (b) CISPH-HS-ASA and CIP (Hu and Kashiwagi, 2004)

Fig. 10. Efficient removal of false zero-pressure points at the wall boundaries in the dam break simulation by CISPH-HS-ASA method - snapshots by CISPH-HS and CISPH-HS-ASA methods at $t=0.750 \mathrm{~s}$

Fig. 11. Dam break simulation (Hu and Kashiwagi, 2004) - Snapshots of water particles together with pressure field at $t=0.750 \mathrm{~s}$ by CISPH-HS and CISPH-HS-ASA methods with different initial particle spacings

Fig. 12 Dam break simulation (Hu and Kashiwagi, 2004) - Time history of calculated pressure at measuring point A corresponding to (a) CISPH-HS-ASA simulations with different initial particle spacings (b) CISPH-HS and CISPH-HS-ASA simulations with initial particle spacings of $d_{0}=0.006 \mathrm{~m}$ and $d_{0}=0.002 \mathrm{~m}$ 
Fig. 13. Non-Symmetric (a) and almost symmetric (b) distribution of neighboring particles in a particle-based calculation

Fig. 14. A schematic sketch of the dam break simulation corresponding to the experiment by Zhou et al. (1999)

Fig. 15. Dam break simulation (Zhou et al., 1999) - Snapshots by CISPH-HS-ASA illustrating (a) the development of the reversing jet (b) the impact of reversing jet and (c) the development of splash-up

Fig. 16. Dam break simulation (Zhou et al., 1999) - qualitative comparison between ISPH and improved ISPH methods

Fig. 17. Dam break simulation (Zhou et al., 1999) - comparison between the results by ISPH and improved ISPH methods and those by improved WCSPH (Colagrossi and Landrini, 2003) and BEM (Greco et al., 2004) methods

Fig. 18. Time history of experimental (Zhou et al., 1999) and calculated pressure at measuring point B comparison between (a) ISPH and improved ISPH (b) CISPH-HS and CISPH-HS-ASA and

(c) CISPH-HS, CISPH-HS-ASA and other numerical methods

Fig. 19. Dam break simulation (Zhou et al., 1999) - time variation of water heights $h_{1}$ and $h_{2}$ at locations (a) $(x / H)_{1}=0.825$ and (b) $(x / H)_{2}=1.653$ from the right vertical wall

Fig. 20. (a) A schematic sketch of the numerical domain and (b) positions of measuring points in a flip-through impact corresponding to the experiment by Hattori et al. (1994)

Fig. 21. (a) ISPH and improved ISPH snapshots of water particles and pressure field during a flip-through impact at $t^{*}=-800$ and $t^{*}=0$

Fig. 21. (b) ISPH and improved ISPH snapshots of water particles and pressure field during a flip-through impact at $t^{*}=200$ and $t^{*}=500$

Fig. 22. A schematic sketch of the pressure contours at the time of maximum impact pressure computed by Cooker and Peregrine (1992)

Fig. 23. Dimensionless calculated and experimental wave pressure at different points along a vertical wall during a flip-through impact (Hattori et al., 1994) 
Table 1. Description of the ISPH and improved ISPH methods applied in this study

\begin{tabular}{|c|c|c|}
\hline Method & Abbreviation & Description \\
\hline Incompressible SPH & ISPH & $\begin{array}{c}\text { Incompressible SPH method } \\
\text { (Shao and Lo, 2003) }\end{array}$ \\
\hline Corrected Incompressible SPH & CISPH & $\begin{array}{c}\text { Incompressible SPH method modified } \\
\text { by Eq. 10 (Khayyer et al., 2008) }\end{array}$ \\
\hline $\begin{array}{c}\text { Incompressible SPH method with a } \\
\text { Higher order Source term }\end{array}$ & ISPH-HS & $\begin{array}{c}\text { Incompressible SPH method modified } \\
\text { by Eq. 16 }\end{array}$ \\
\hline $\begin{array}{c}\text { Corrected Incompressible SPH method } \\
\text { with a Higher order Source term }\end{array}$ & CISPH-HS & $\begin{array}{c}\text { Incompressible SPH method modified } \\
\text { by Eqs. 10 and 16 }\end{array}$ \\
\hline $\begin{array}{c}\text { CISPH-HS - Assessment of free-surface } \\
\text { based on nearly Symmetric Arrangement } \\
\text { of non-free-surface particles }\end{array}$ & CISPH-HS-ASA & $\begin{array}{c}\text { Incompressible SPH method modified } \\
\text { by Eqs. 10, 16 and 19 }\end{array}$ \\
\hline
\end{tabular}

Table 2. Required CPU time per calculation time step for ISPH and improved ISPH simulations of a Dam break with impact (Hu and Kashiwagi, 2004)

\begin{tabular}{|c|c|c|c|c|c|}
\hline Method & ISPH & CISPH & ISPH-HS & CISPH-HS & CISPH-HS-ASA \\
\hline $\begin{array}{c}\text { CPU time per } \\
\text { calculation time step (s) }\end{array}$ & 0.842 & 0.875 & 0.825 & 0.854 & 0.858 \\
\hline
\end{tabular}

Table 3. Required CPU time per calculation time step for CISPH-HS-ASA simulations with different spatial resolutions - Dam break with impact (Hu and Kashiwagi, 2004)

\begin{tabular}{|c|c|c|c|c|c|}
\hline Particle Size (m) & 0.006 & 0.005 & 0.004 & 0.003 & 0.002 \\
\hline $\begin{array}{c}\text { Total Number of } \\
\text { Particles }\end{array}$ & 2524 & 3720 & 5466 & 9566 & 21132 \\
\hline $\begin{array}{c}\text { CPU time per } \\
\text { calculation time step (s) }\end{array}$ & 0.125 & 0.314 & 0.858 & 1.906 & 5.156 \\
\hline
\end{tabular}




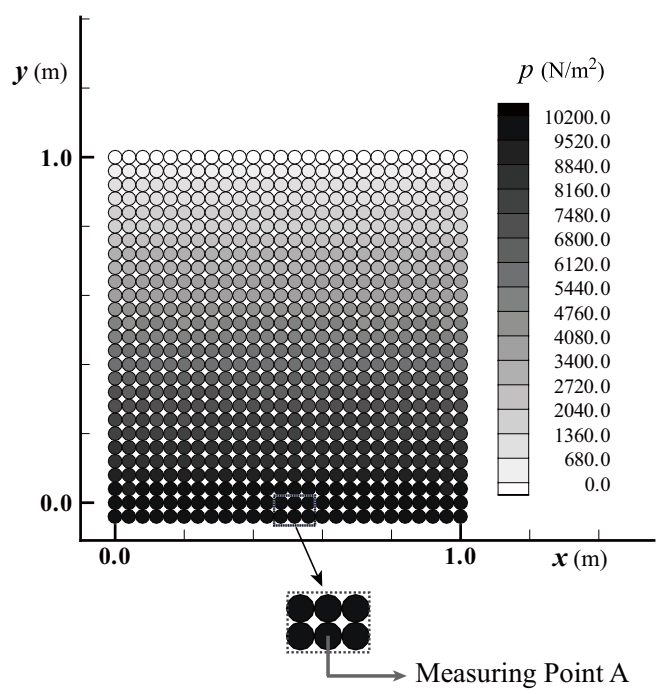

Fig. 1. A schematic sketch of the computational domain for hydrostatic pressure calculation

(a1)

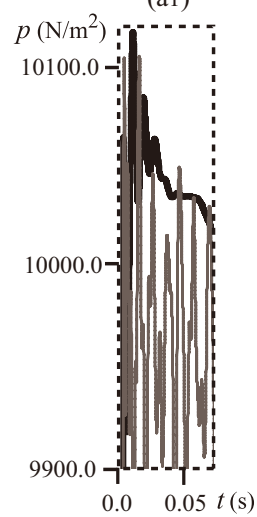

10200.0

$p\left(\mathrm{~N} / \mathrm{m}^{2}\right)$

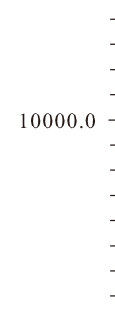

9800.0

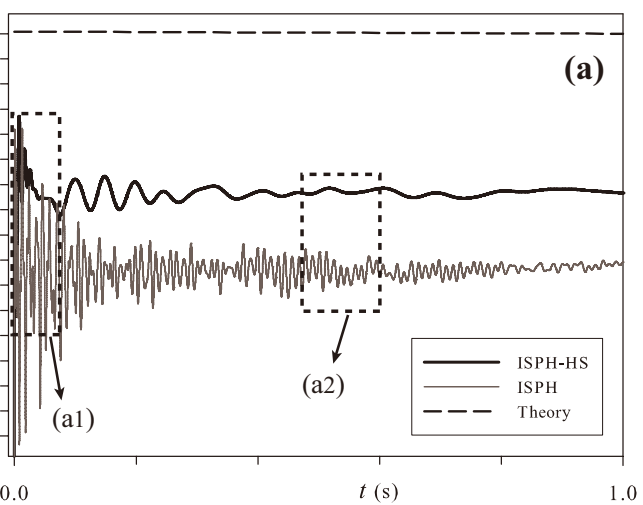

(a2)

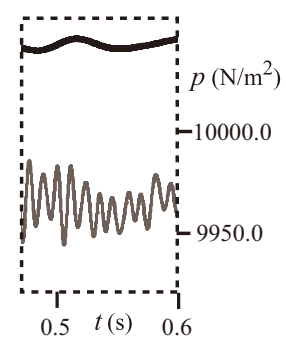

(b2)

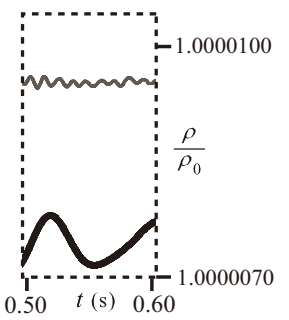

$\frac{\rho}{\rho_{0}}$
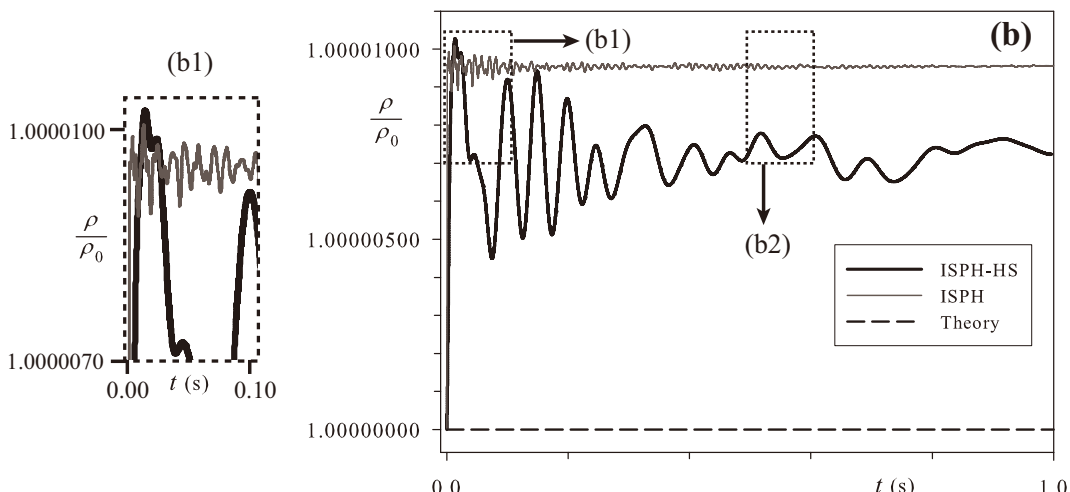

$0.50 \quad t(\mathrm{~s}) \quad 0.60$

0.0

$t(\mathrm{~s})$

1.0

Fig. 2. Hydrostatic pressure calculation - time variation of (a) pressure and (b) density at measuring point $\mathrm{A}$ 
(a1)

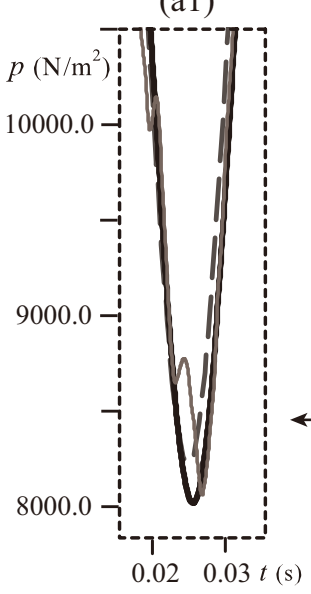

(a)

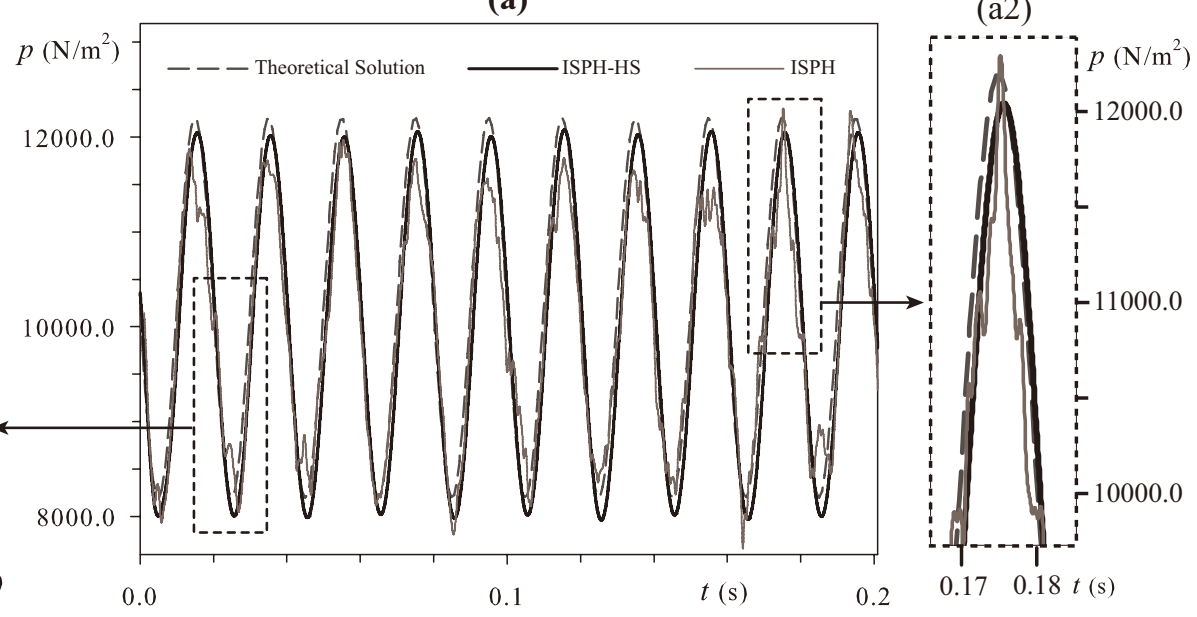

(b)
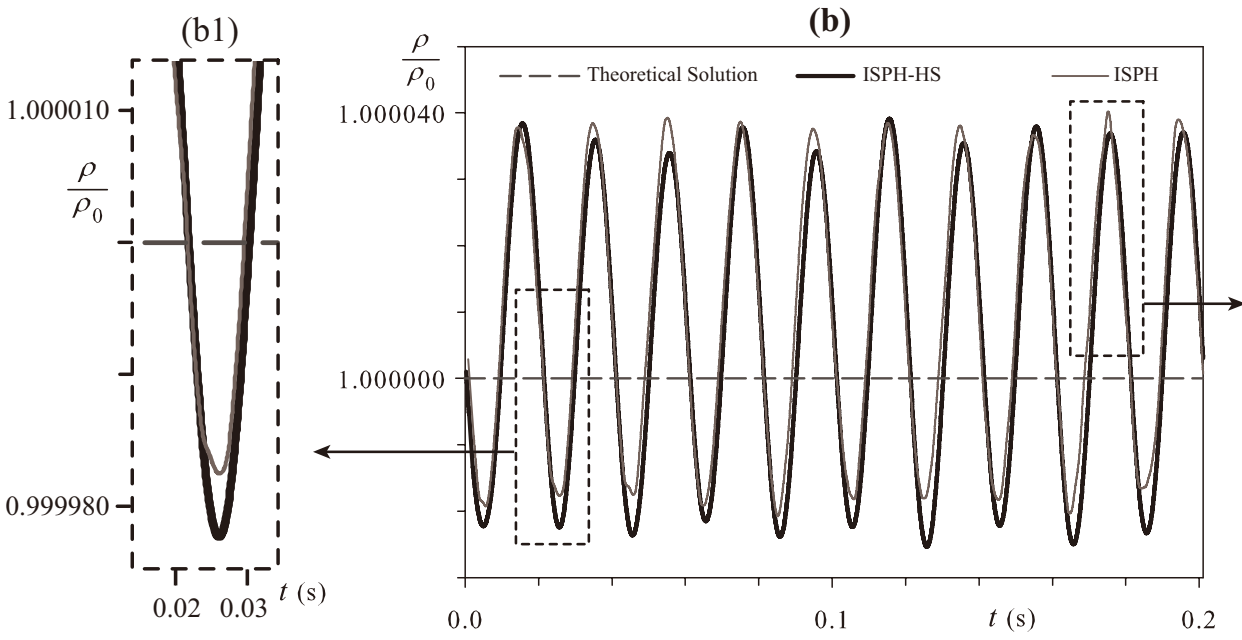

(b2)

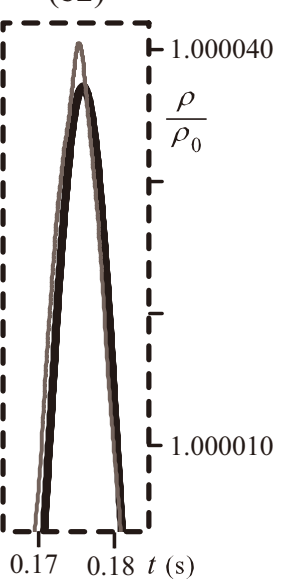

Fig. 3. Time variation of (a) pressure and (b) density at measuring point $A$ in the hydrostatic pressure calculation plus a designed sinusoidal variation of source term of Poisson Pressure Equation

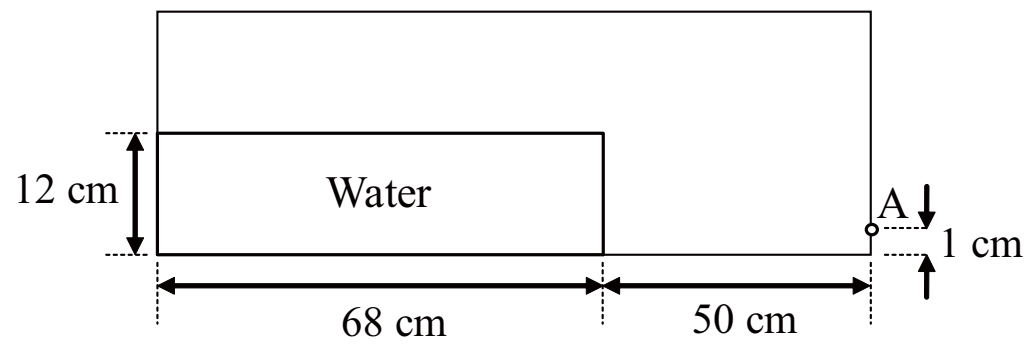

Fig. 4. A schematic sketch of the dam break simulation corresponding to the experiment by Hu and Kashiwagi (2004) 

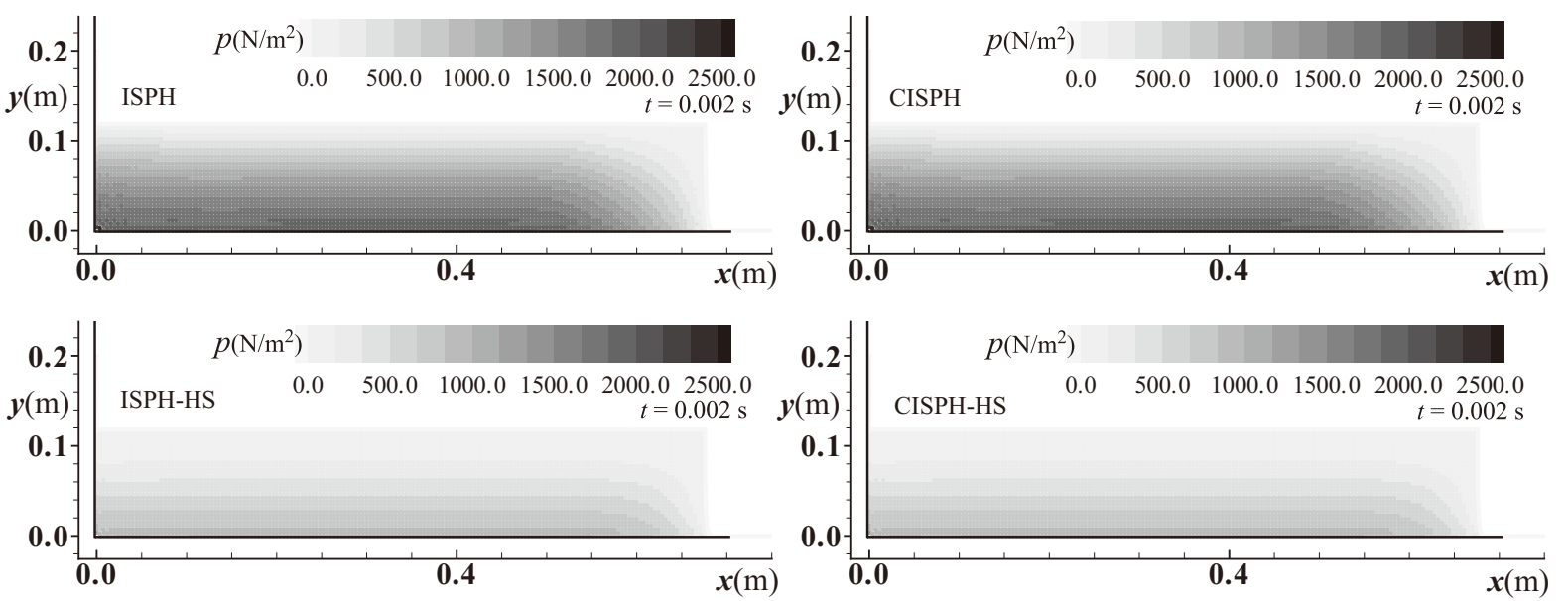

Fig. 5. Dam break simulation (Hu and Kashiwagi, 2004) - Snapshots of water particles at $t=0.002 \mathrm{~s}$
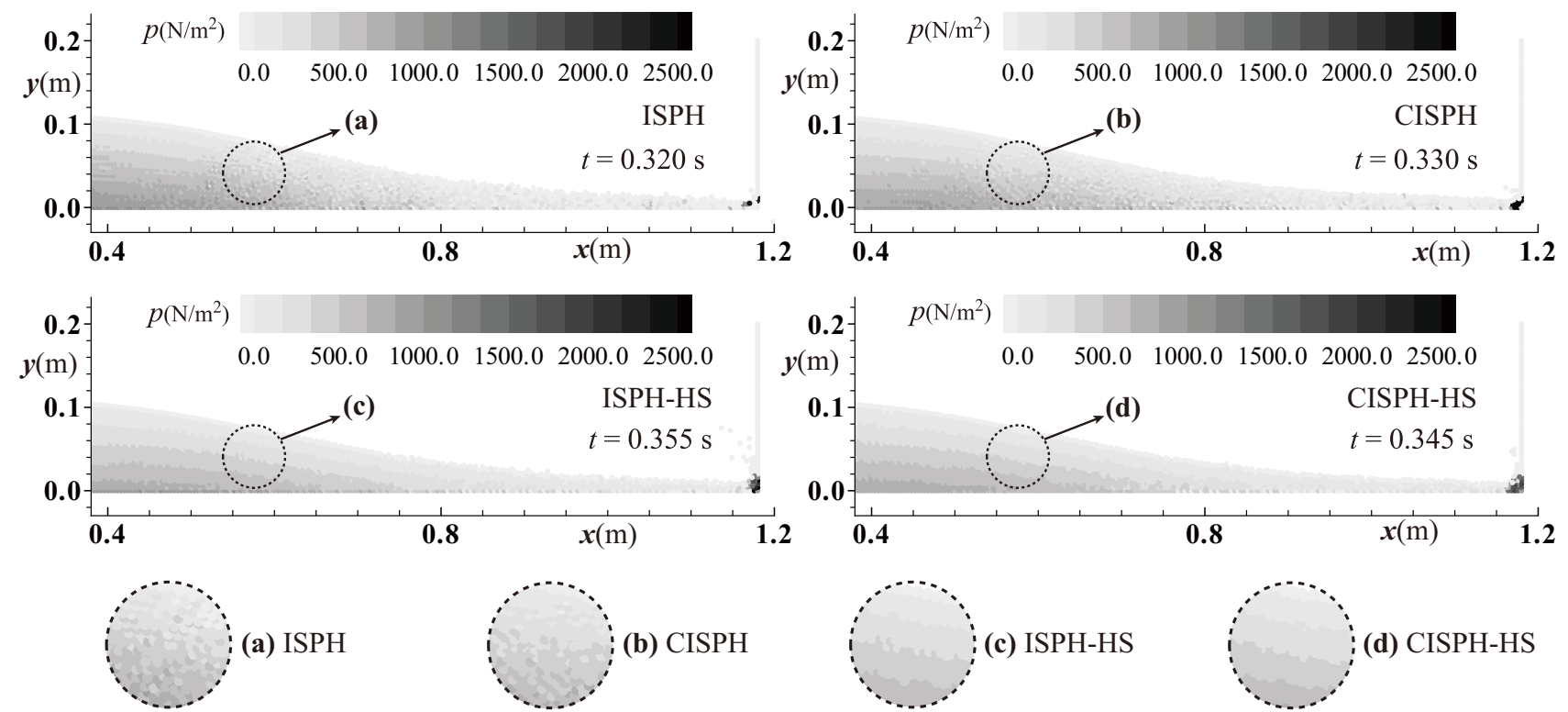

(a) ISPH
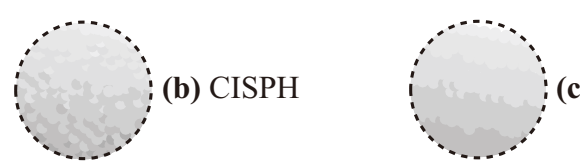

(c) ISPH-HS

Fig. 6. Dam break simulation (Hu and Kashiwagi, 2004) - Snapshots of water particles at the impact instant when the maximum impact pressure is recorded 

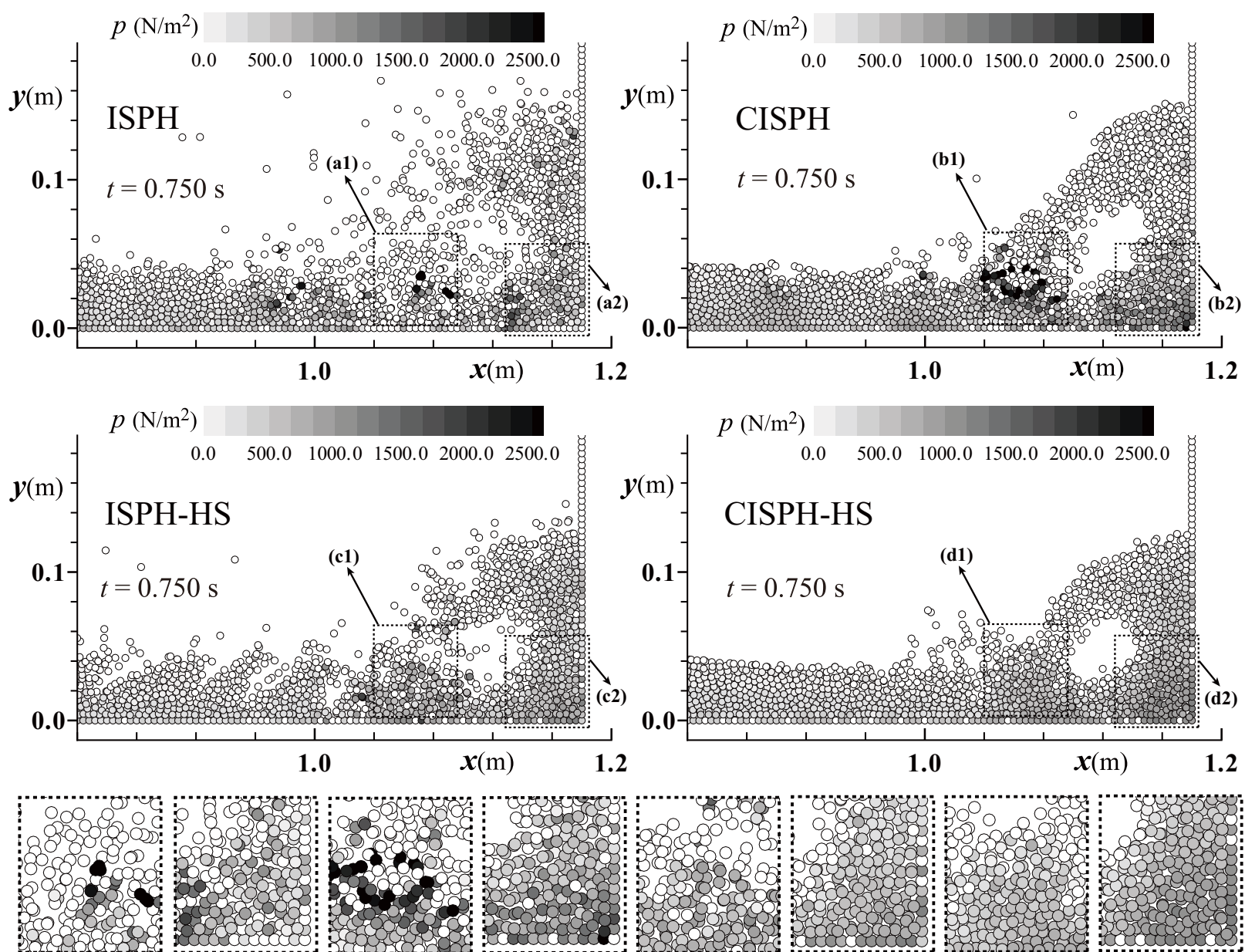

(a1) ISPH

(a2) ISPH

(b1) CISPH
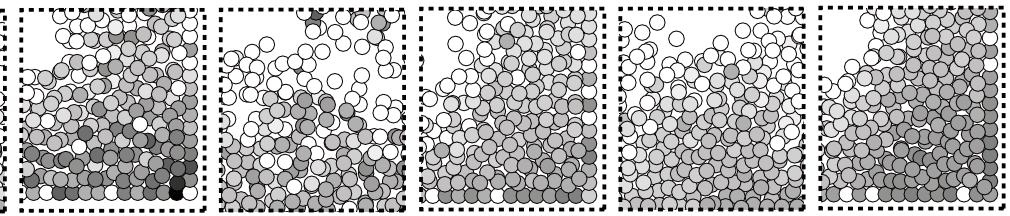

Fig. 7. Dam break simulation (Hu and Kashiwagi, 2004) - Snapshots of water particles at $t=0.75 \mathrm{~s}$

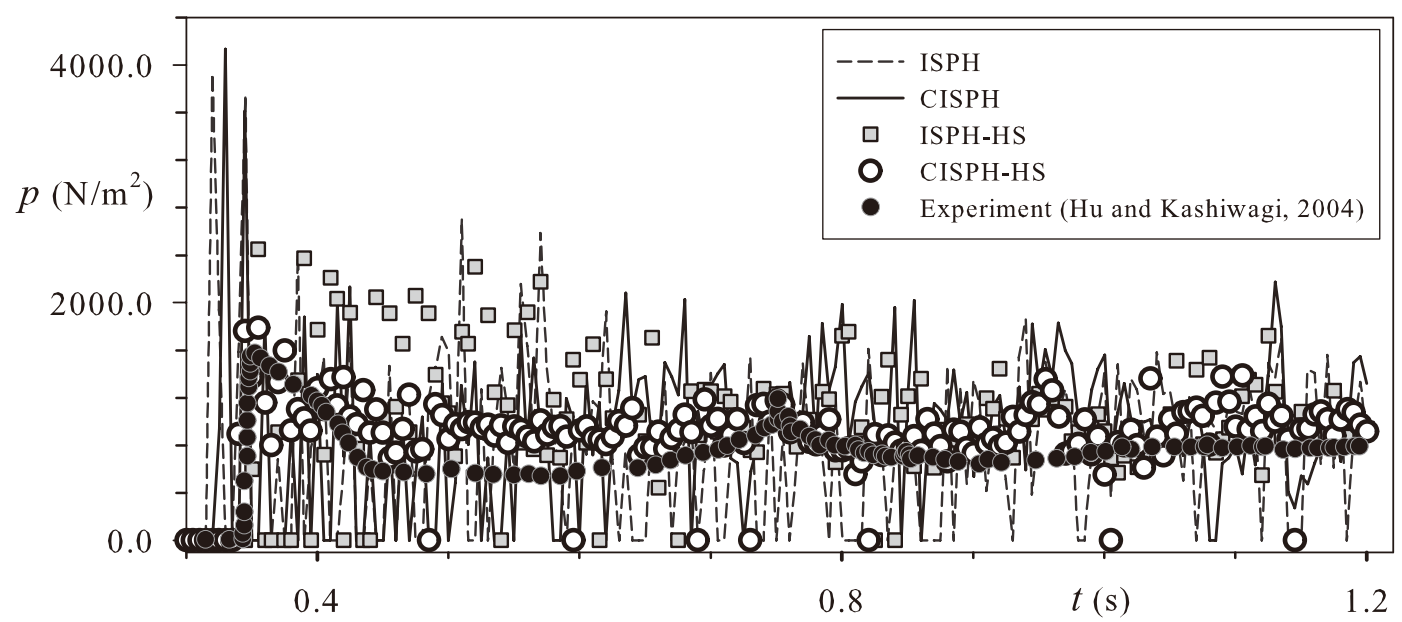

Fig. 8. Dam break simulation (Hu and Kashiwagi, 2004) - time histories of calculated pressure at point A by ISPH and improved ISPH methods 

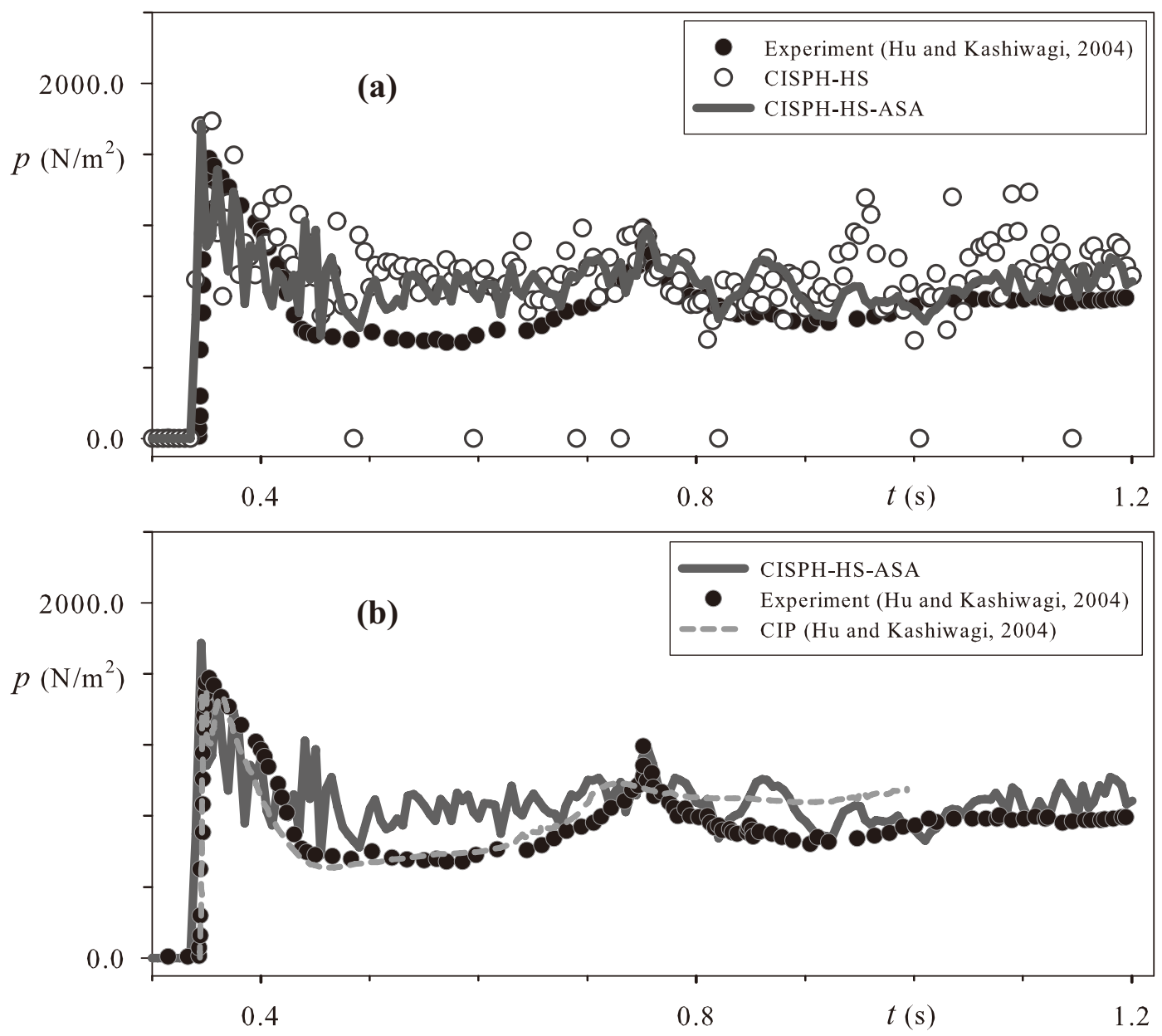

Fig. 9. Dam break simulation - Comparison between the experimental data (Hu and Kashiwagi, 2004), (a) CISPH-HS and CISPH-HS-ASA, (b) CISPH-HS-ASA and CIP (Hu and Kashiwagi, 2004) 


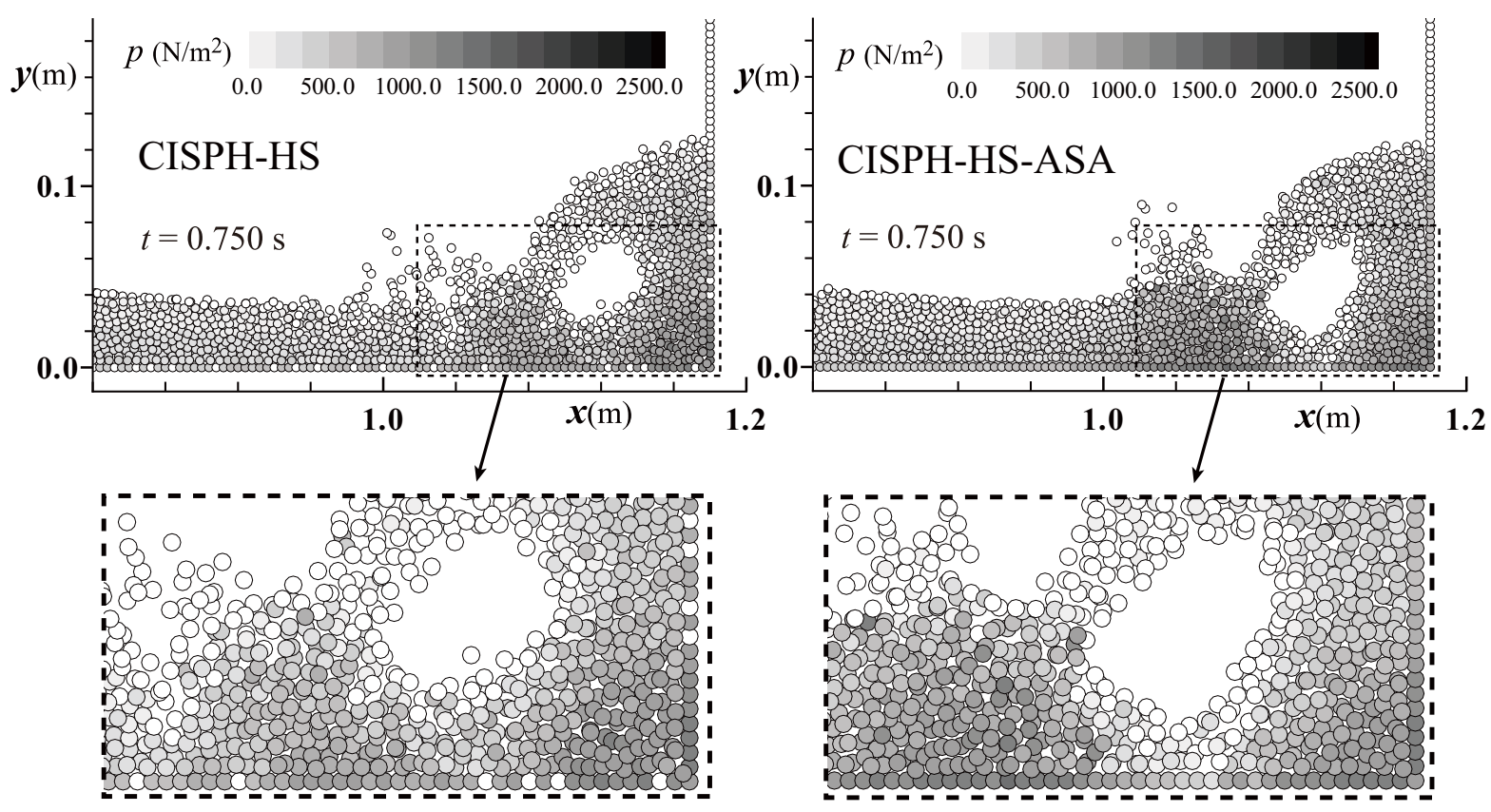

Fig. 10. Efficient removal of false zero-pressure points at the wall boundaries in the dam break simulation by CISPH-HS-ASA method - snapshots by CISPH-HS and CISPH-HS-ASA methods at $t=0.750 \mathrm{~s}$ 

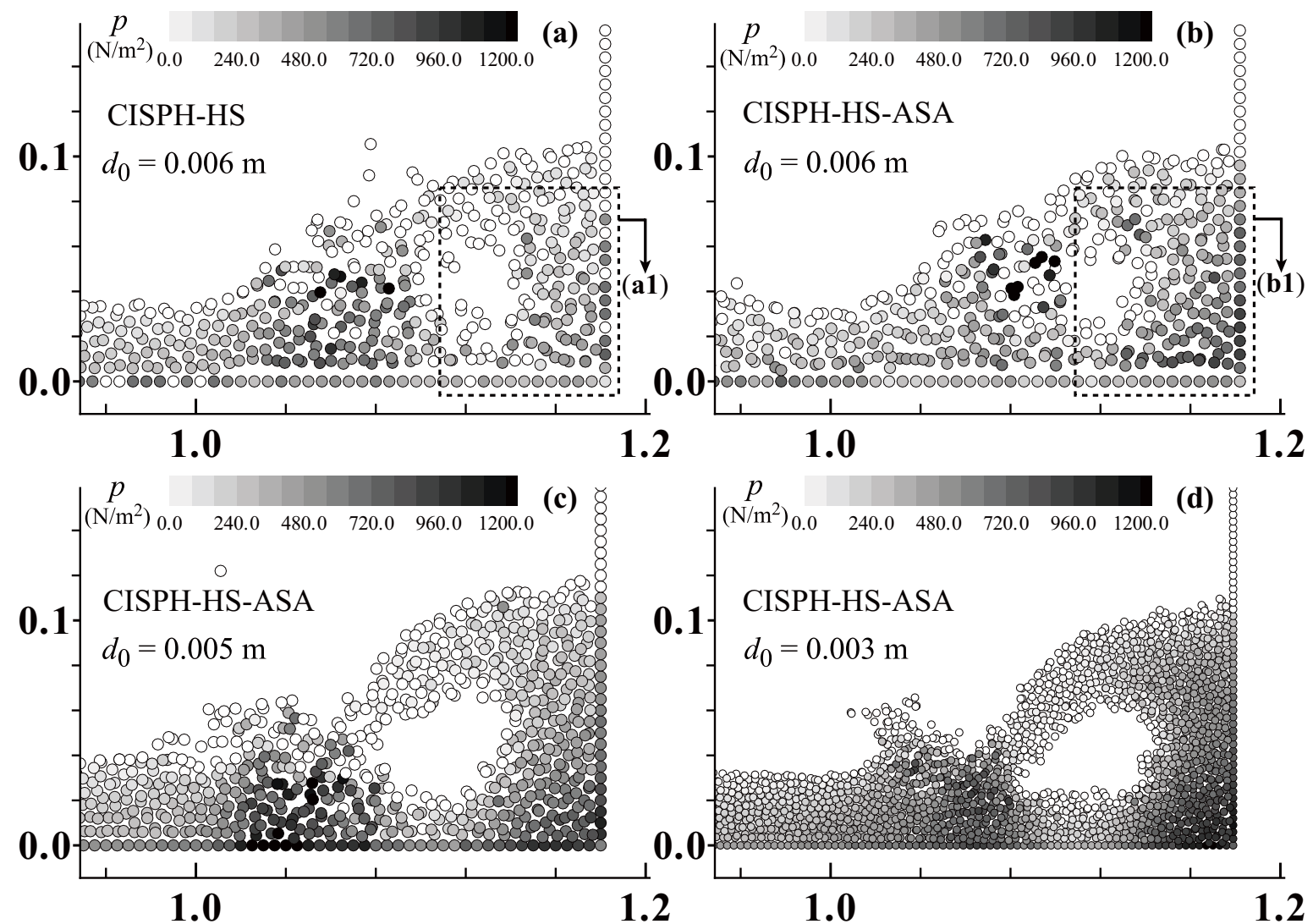

1.2

1.0

1.2

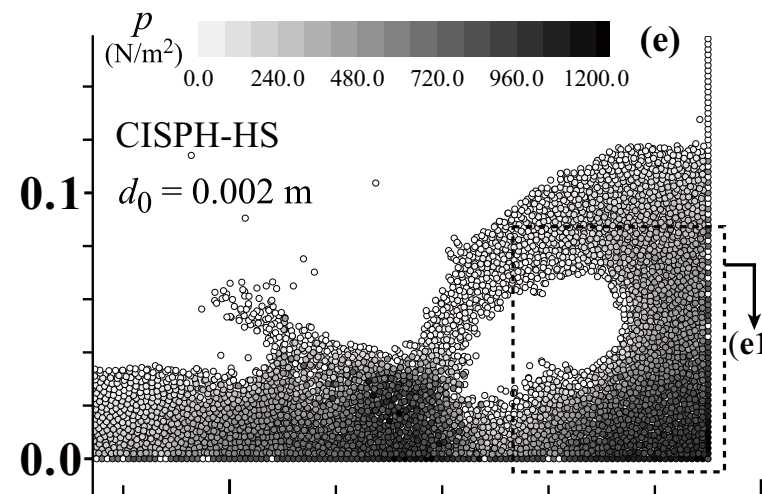

1.2
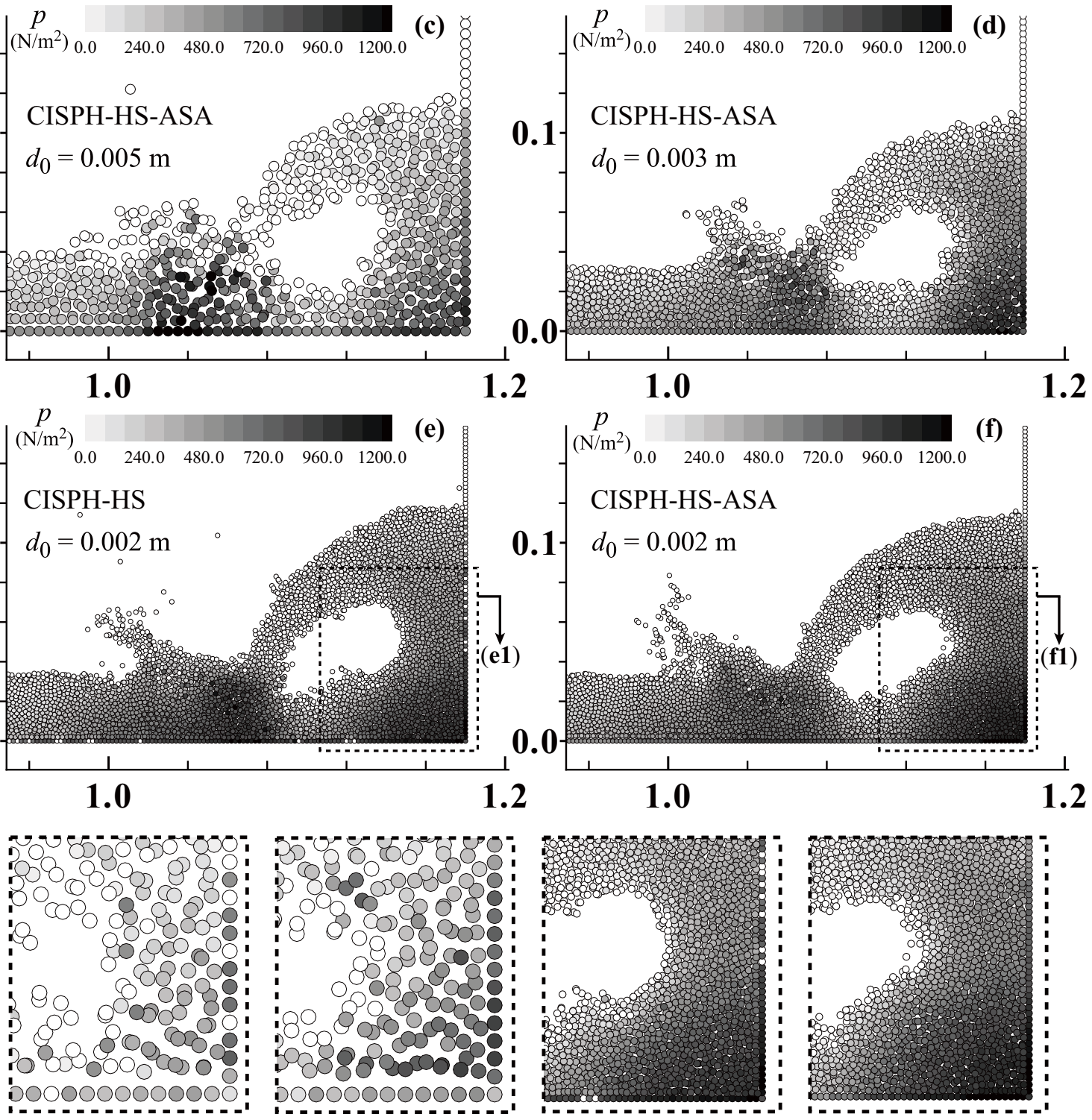

(a1) CISPH-HS

(b1) CISPH-HS-ASA

(e1) CISPH-HS

(f1) CISPH-HS-ASA

Fig. 11. Dam break simulation (Hu and Kashiwagi, 2004) - Snapshots of water particles together with pressure field at $t=0.750 \mathrm{~s}$ by CISPH-HS and CISPH-HS-ASA methods with different initial particle spacings 

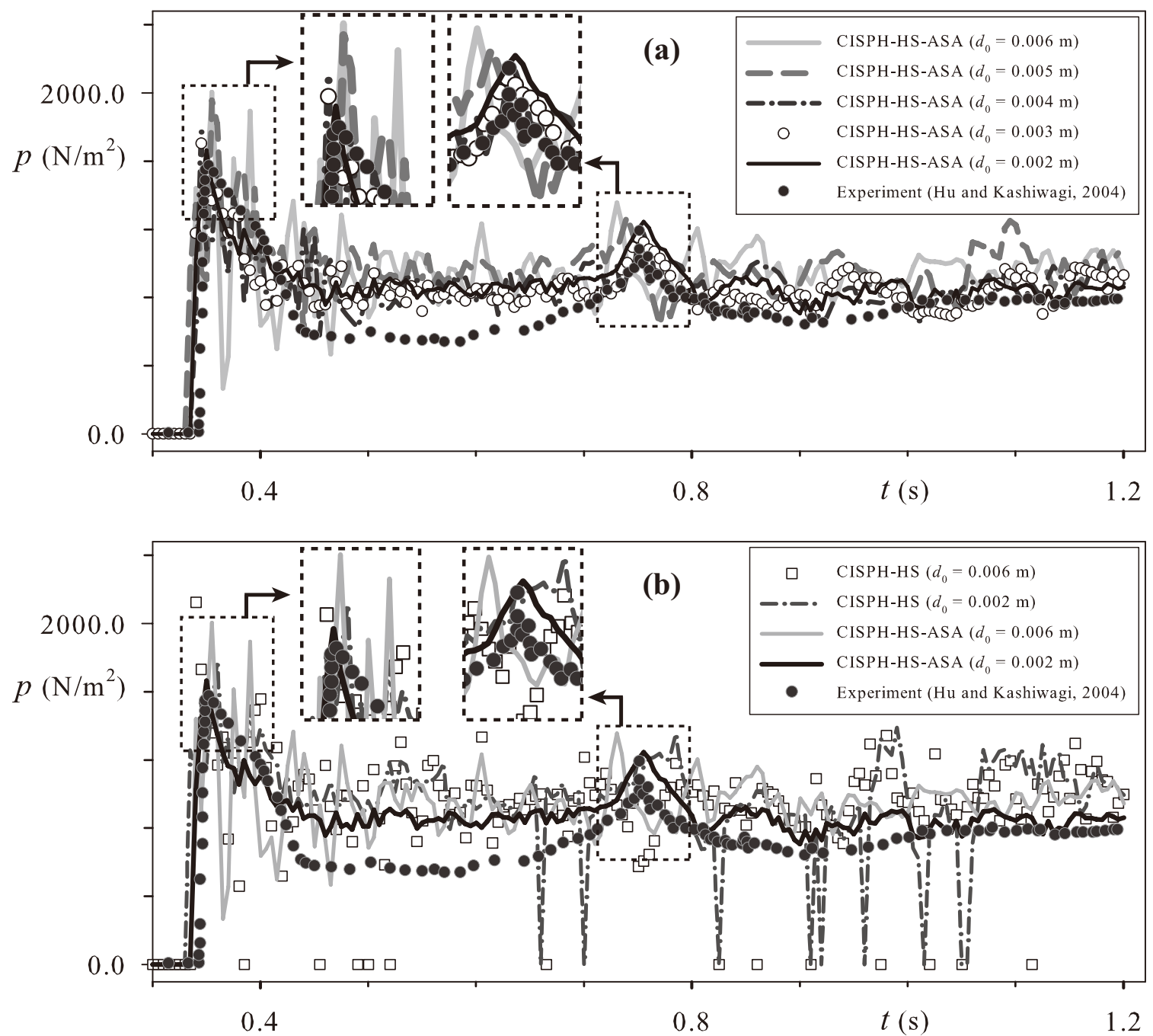

Fig. 12 Dam break simulation (Hu and Kashiwagi, 2004) - Time history of calculated pressure at measuring point A corresponding to (a) CISPH-HS-ASA simulations with different initial particle spacings (b) CISPH-HS and CISPH-HS-ASA simulations with initial particle spacings of $d_{0}=0.006 \mathrm{~m}$ and $d_{0}=0.002 \mathrm{~m}$
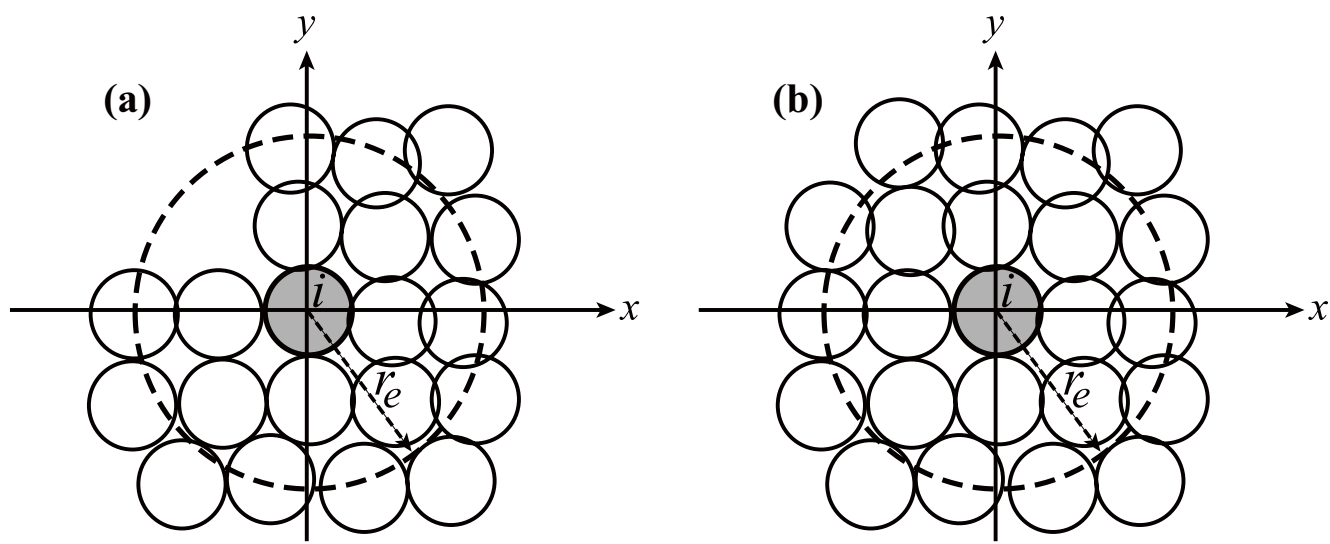

Fig. 13. Non-Symmetric (a) and almost symmetric (b) distribution of neighboring particles in a particle-based calculation 


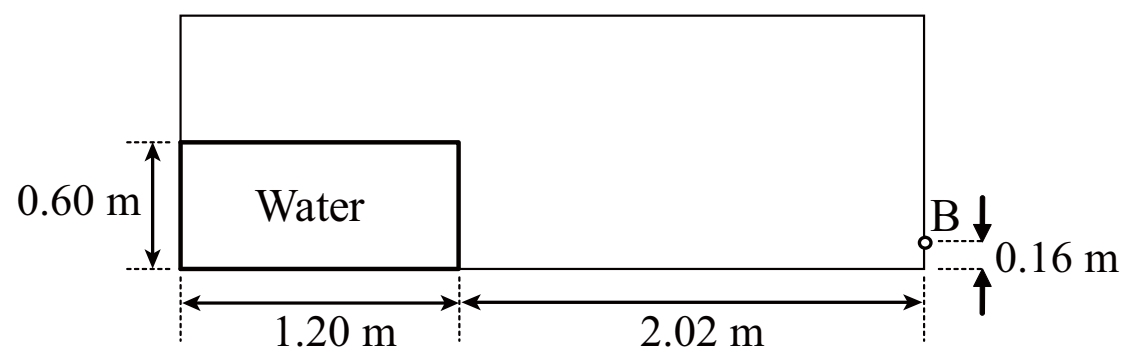

Fig. 14. A schematic sketch of the dam break simulation corresponding to the experiment by Zhou et al. (1999)

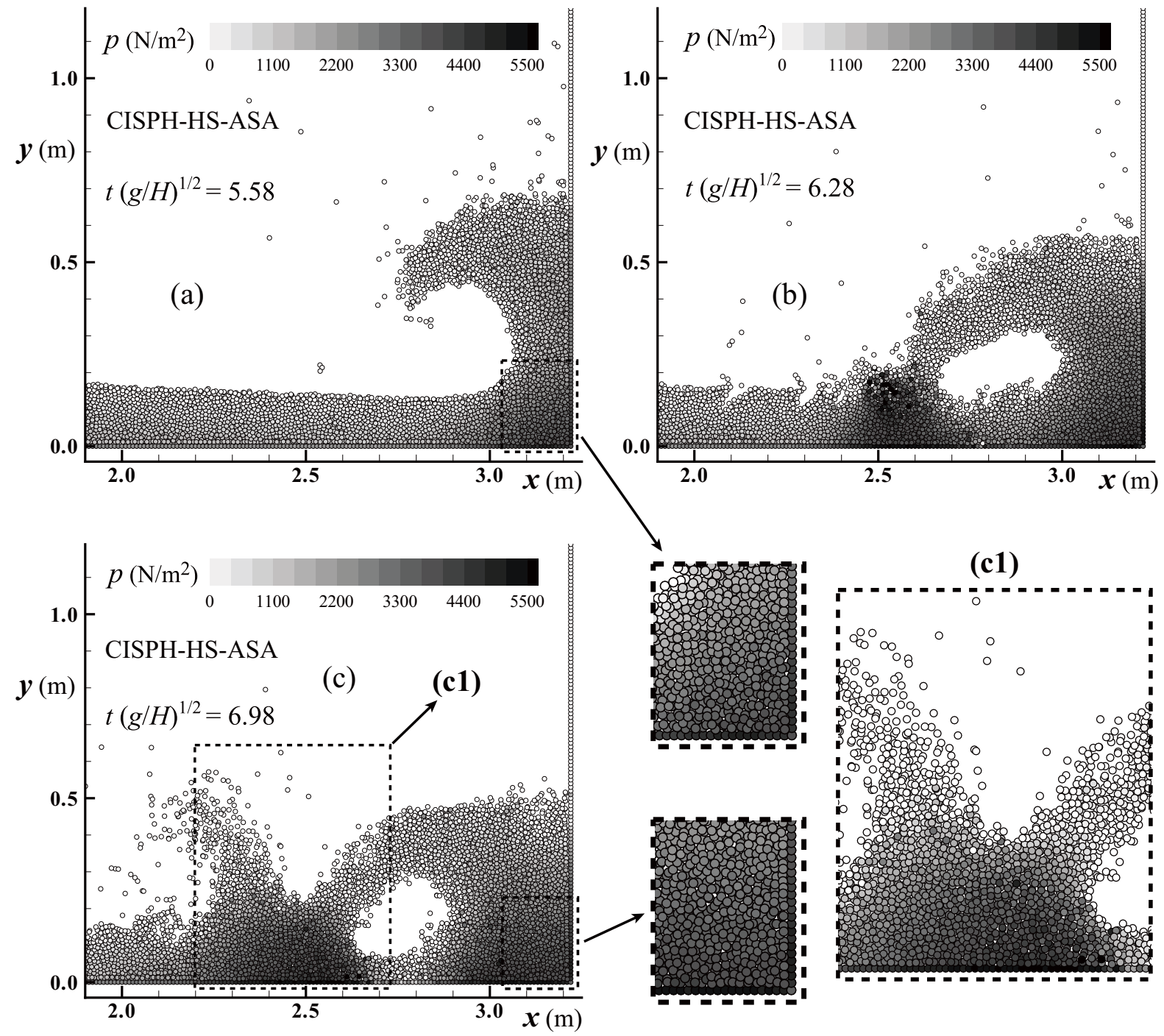

Fig. 15. Dam break simulation (Zhou et al., 1999) - Snapshots by CISPH-HS-ASA illustrating (a) the development of the reversing jet (b) the impact of reversing jet and (c) the development of splash-up 

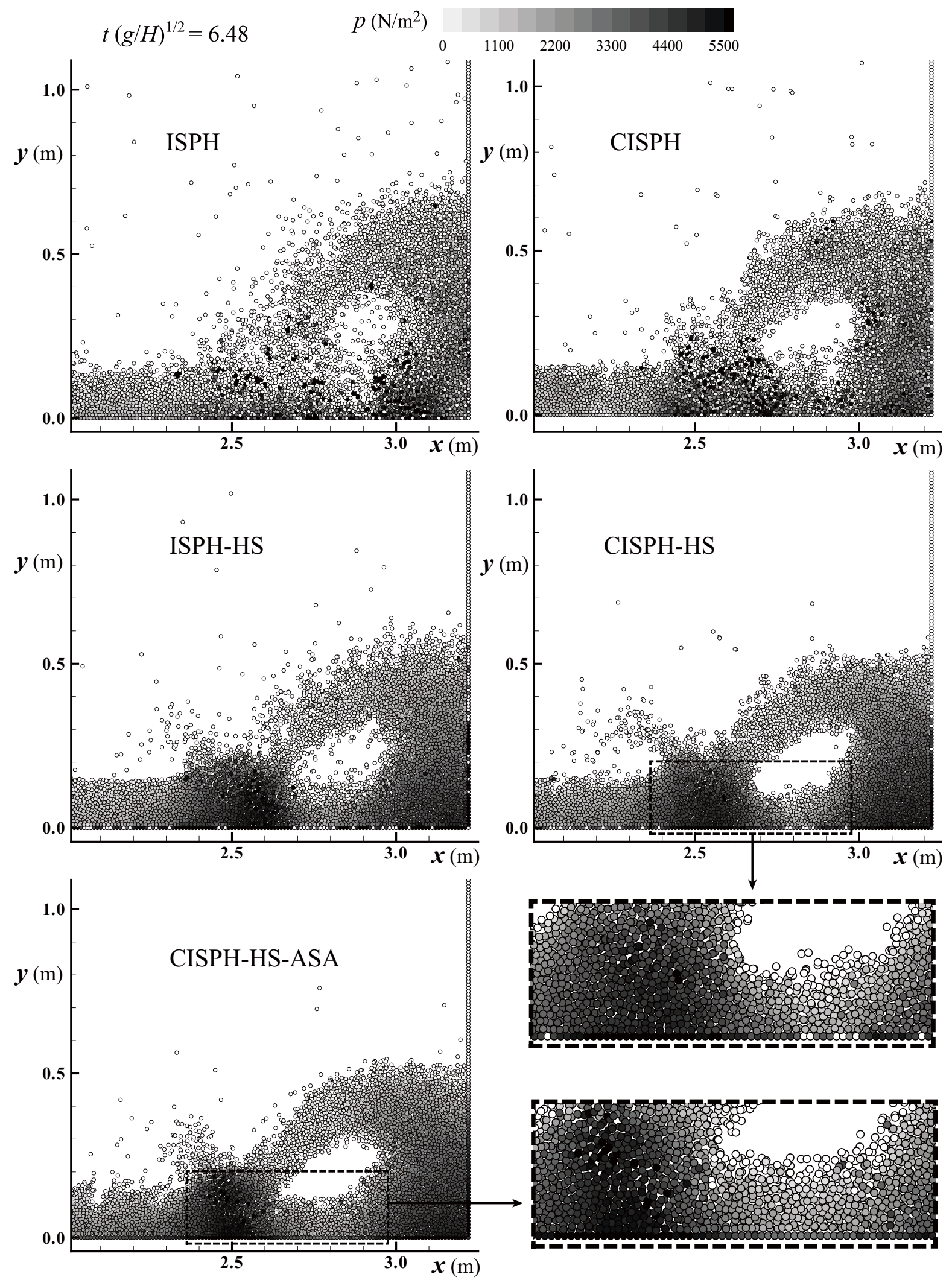

Fig. 16. Dam break simulation (Zhou et al., 1999) - qualitative comparison between ISPH and improved ISPH methods 
$\checkmark \operatorname{BEM}$ (Greco et al., 2004)

- Modified WCSPH (Colagrossi and Landrini, 2003)
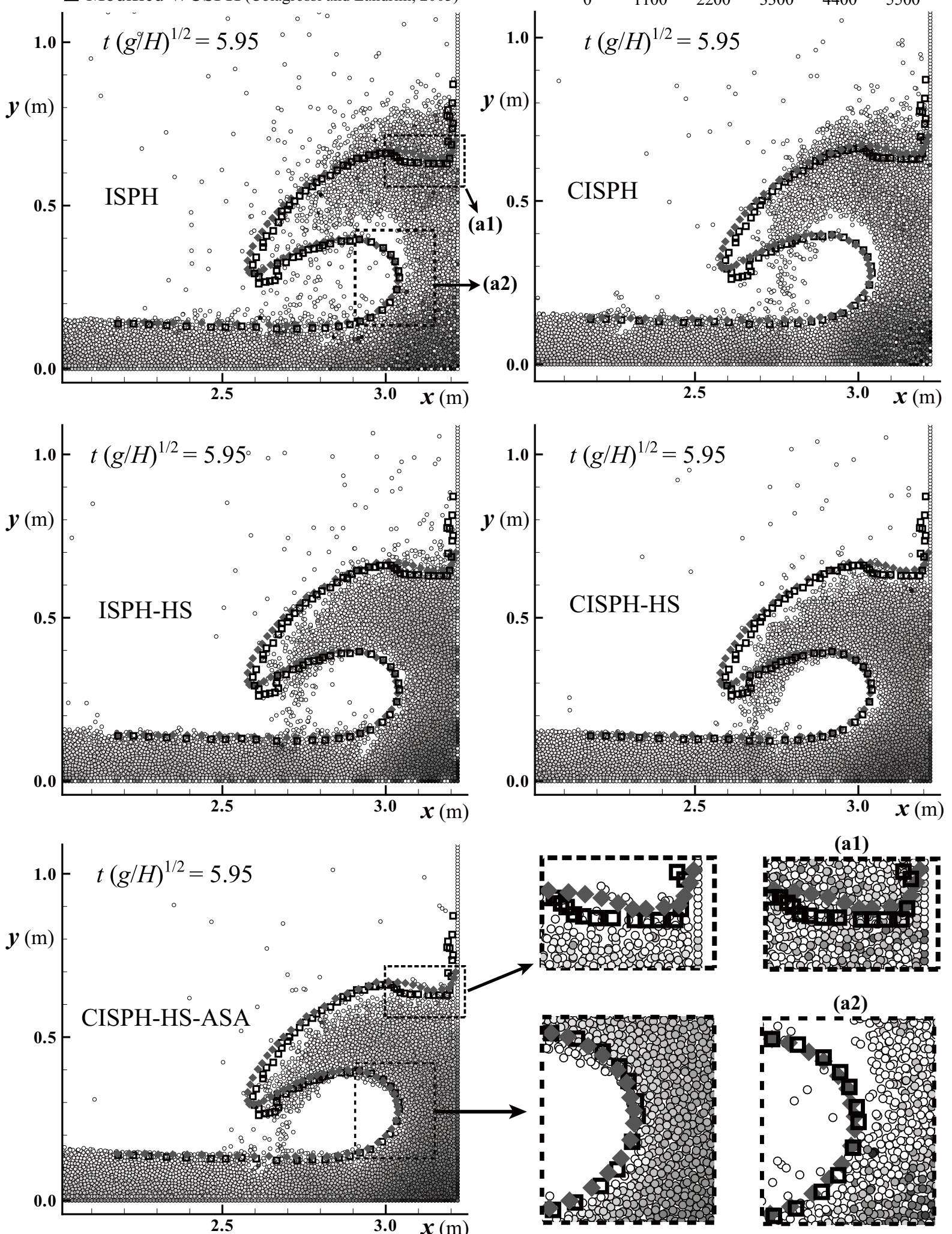

(a1)

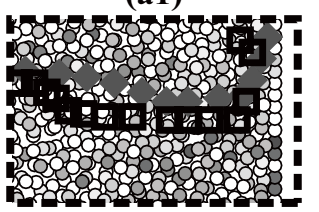

(a2)

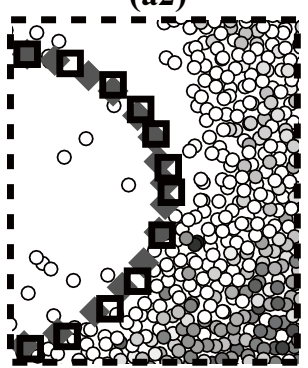

Fig. 17. Dam break simulation (Zhou et al., 1999) - comparison between the results by ISPH and improved ISPH methods and those by improved WCSPH (Colagrossi and Landrini, 2003) and BEM (Greco et al., 2004) methods 

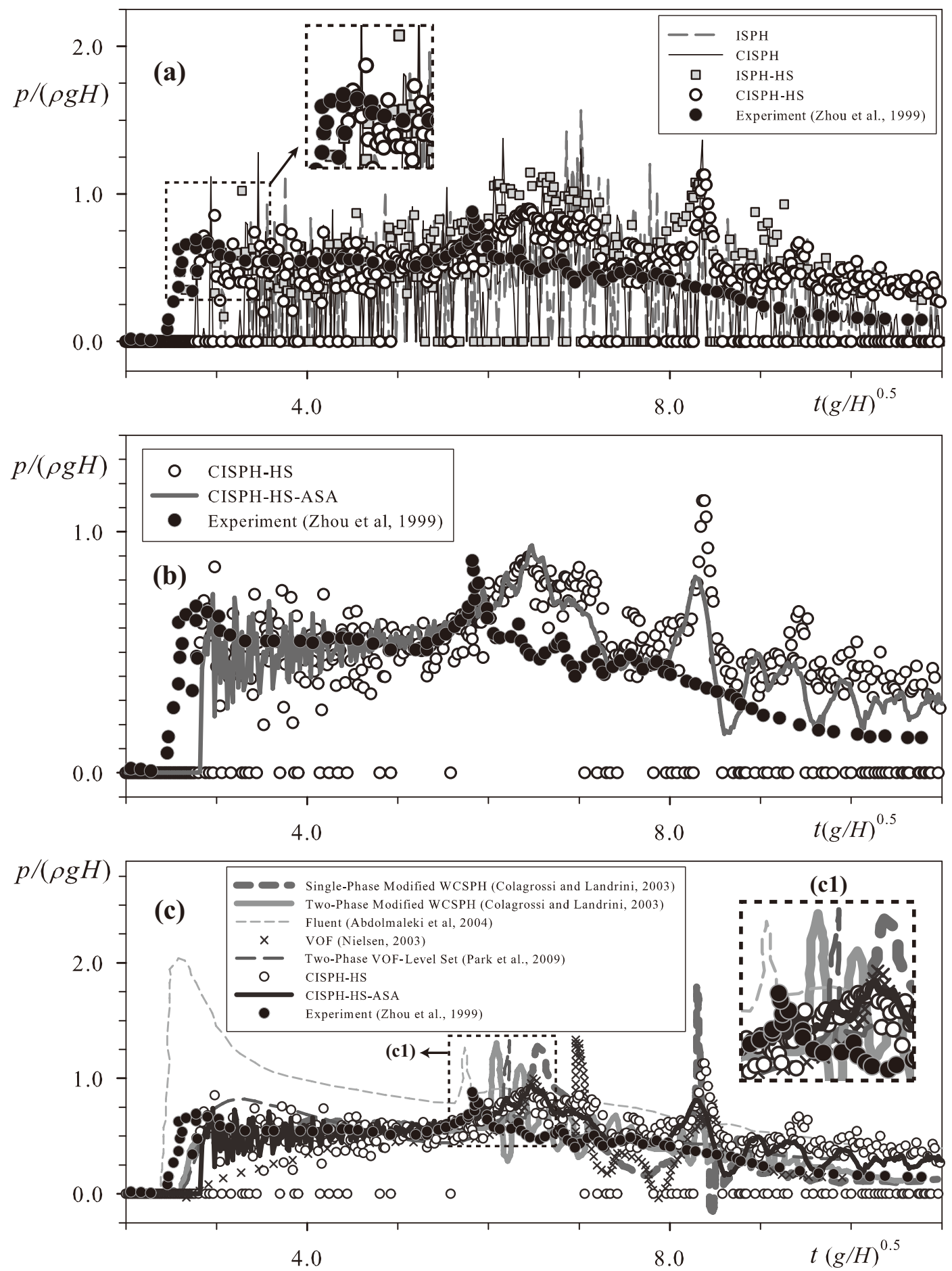

Fig. 18. Time history of experimental (Zhou et al., 1999) and calculated pressure at measuring point B comparison between (a) ISPH and improved ISPH (b) CISPH-HS and CISPH-HS-ASA and (c) CISPH-HS, CISPH-HS-ASA and other numerical methods 

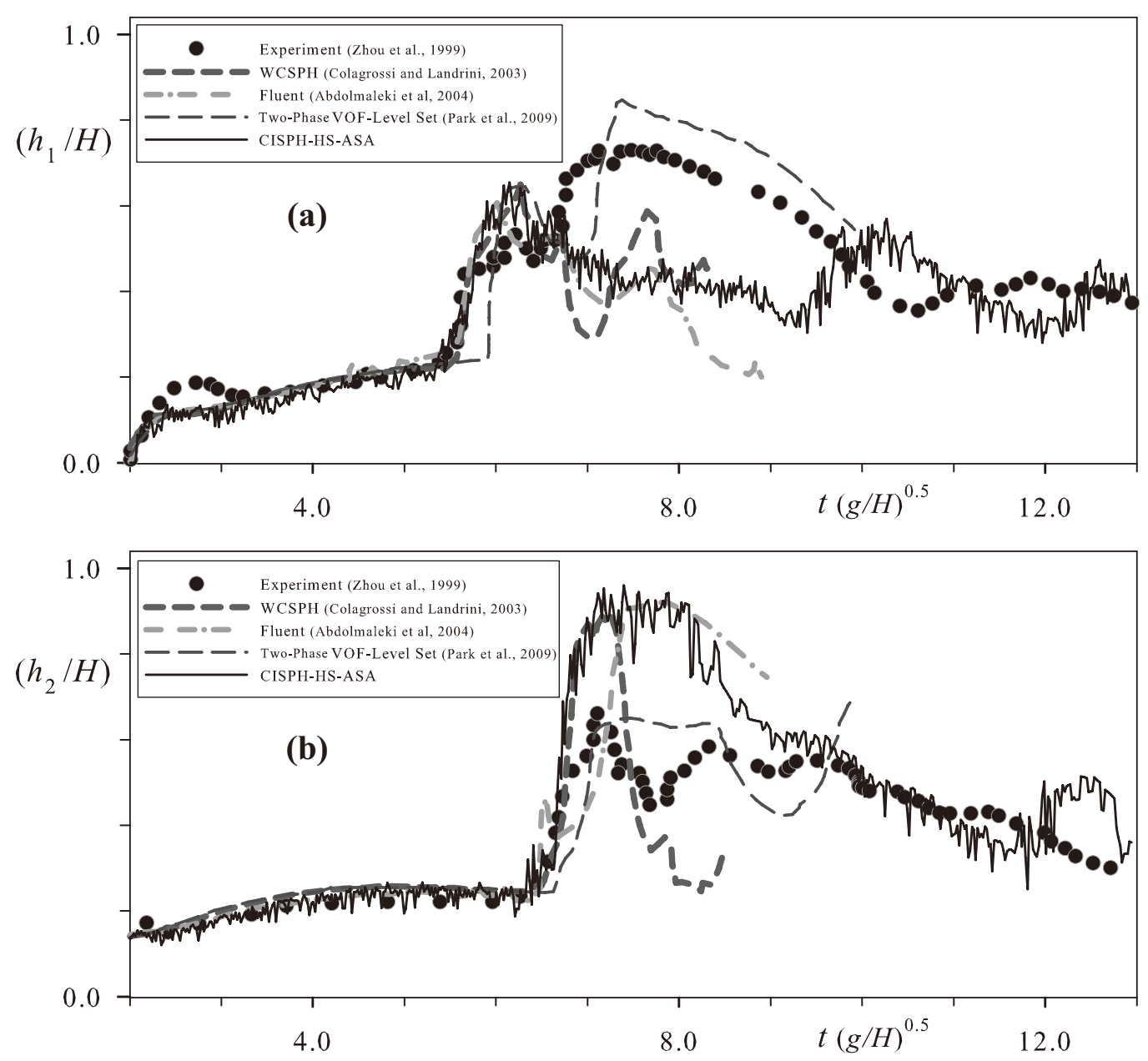

Fig. 19. Dam break simulation (Zhou et al., 1999) - time variation of water heights $h_{1}$ and $h_{2}$ at locations (a) $(x / H)_{1}=0.825$ and (b) $(x / H)_{2}=1.653$ from the right vertical wall
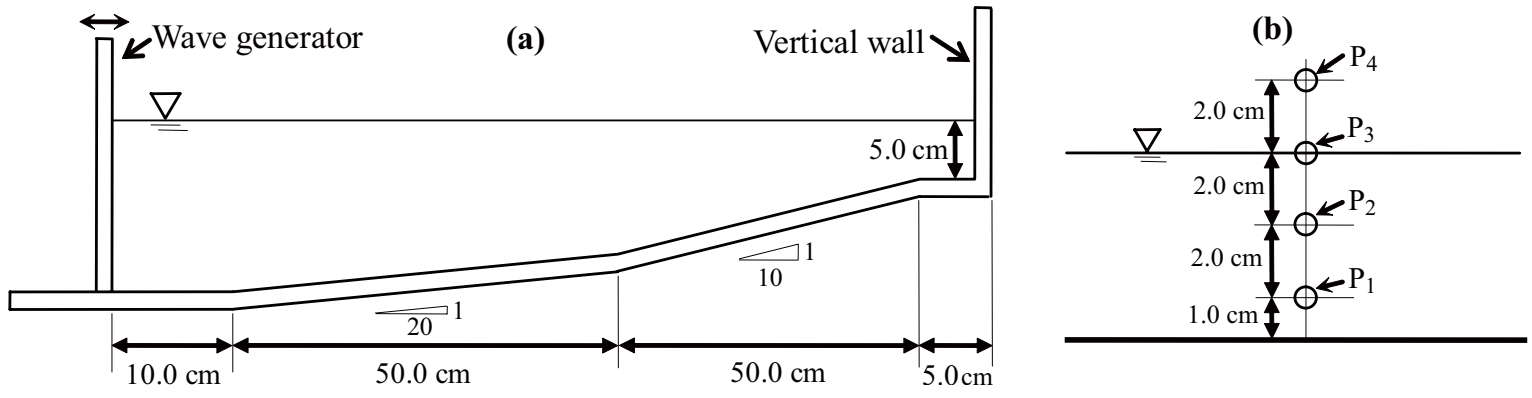

Fig. 20. (a) A schematic sketch of the numerical domain and (b) positions of measuring points in a flip-through impact corresponding to the experiment by Hattori et al. (1994) 

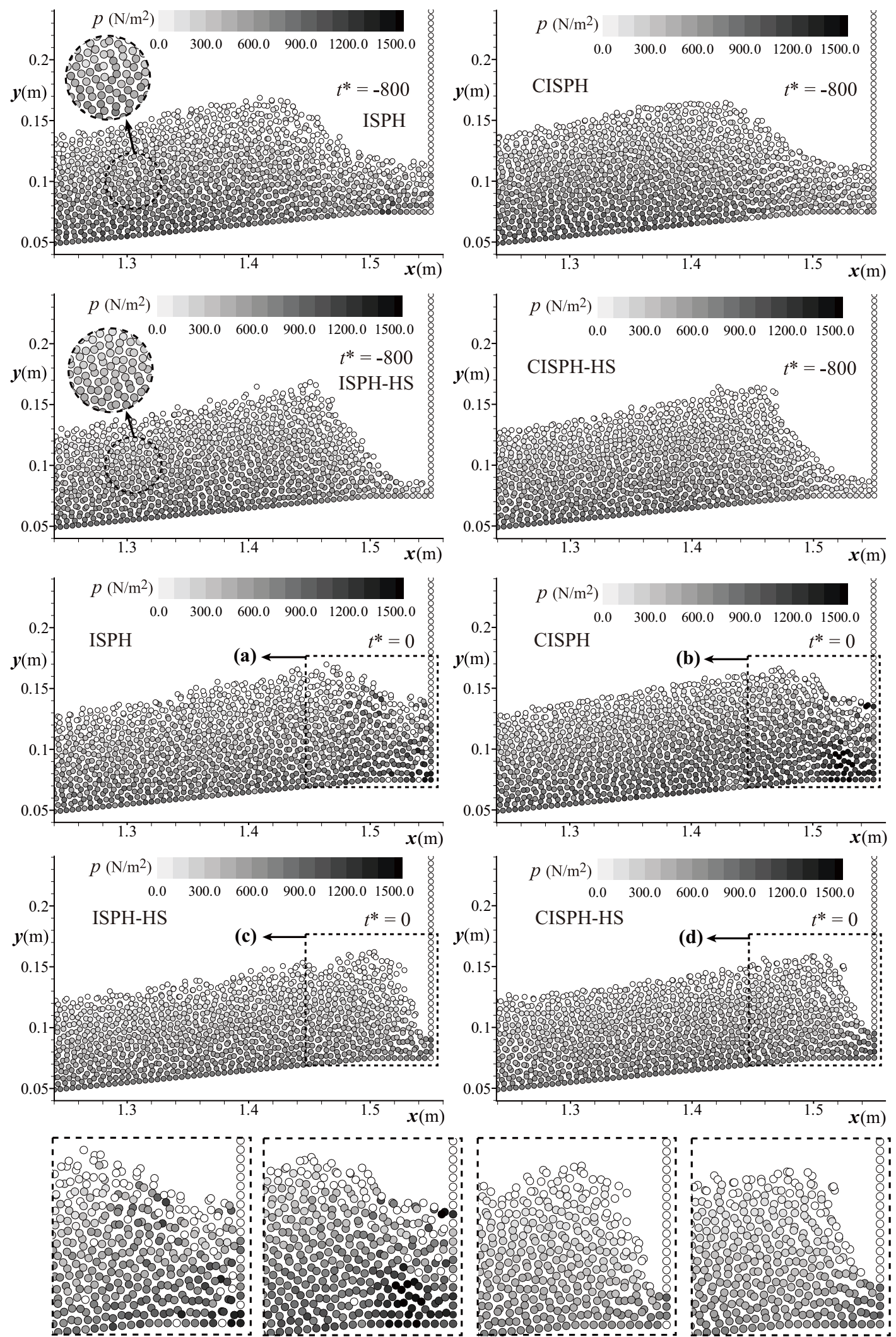

(a) ISPH

(b) CISPH

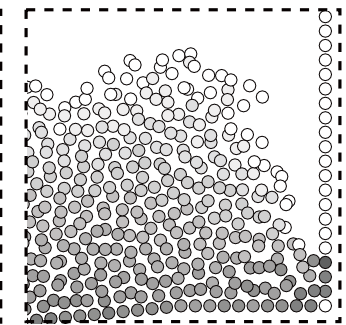

(c) ISPH-HS

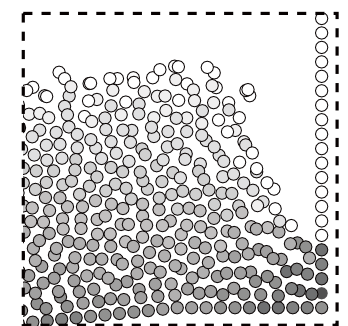

(d) CISPH-HS

Fig. 21. (a) ISPH and improved ISPH snapshots of water particles and pressure field during a flip-through impact at $t^{*}=-800$ and $t^{*}=0$ 

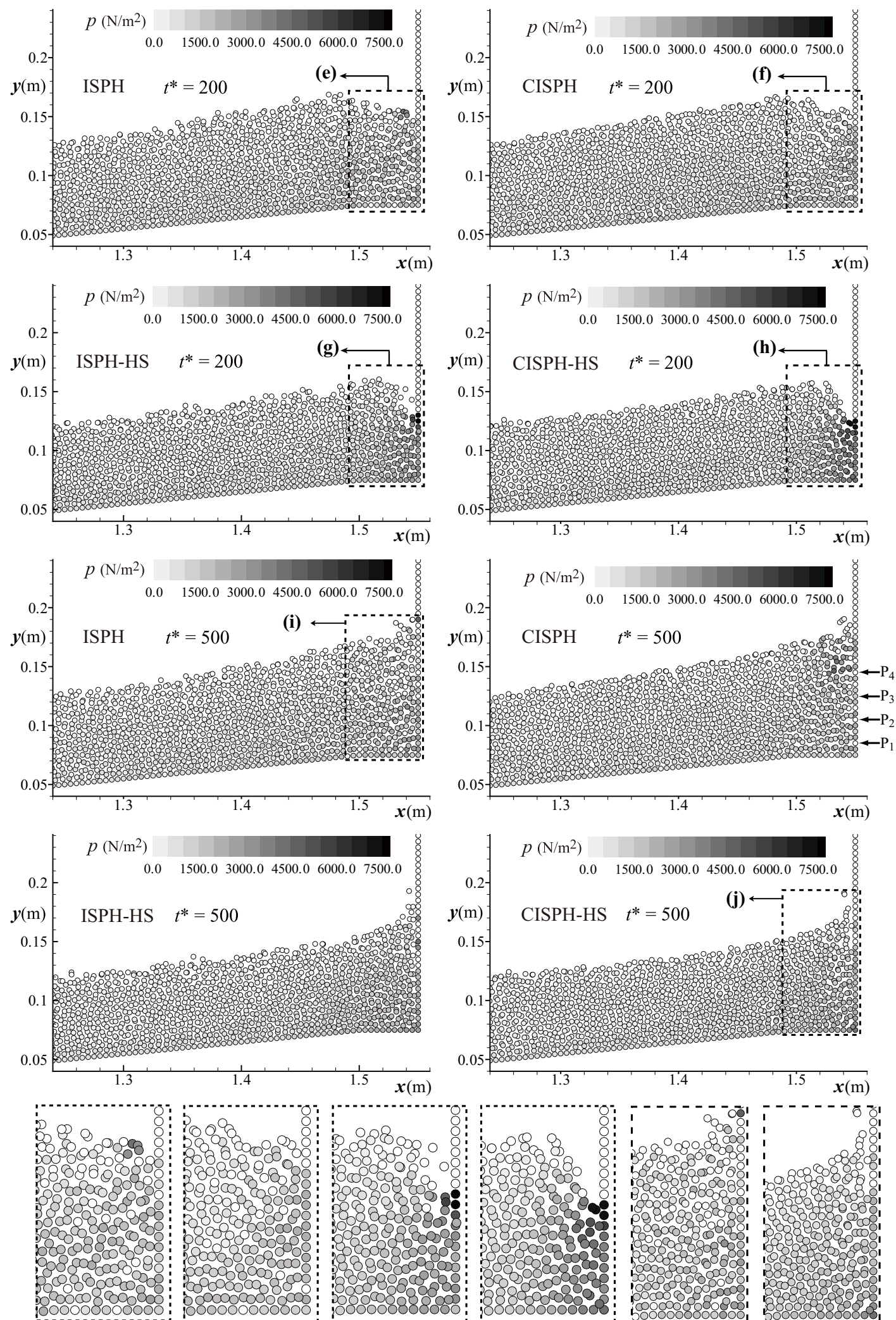

(e) ISPH

(f) $\mathrm{CISPH}$

(g) ISPH-HS
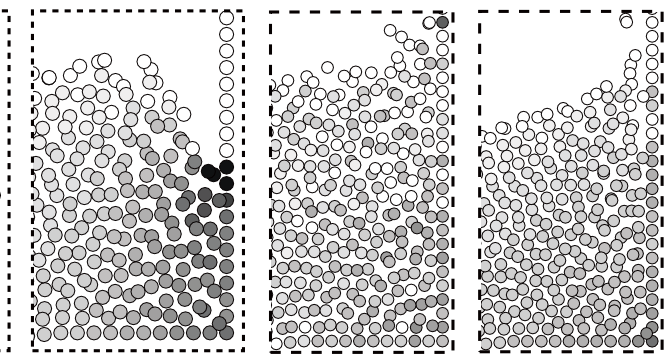

(h) CISPH-HS

(i) $\mathrm{IS} \overline{\mathrm{S}} \overline{\mathrm{H}}$

(j) $\mathrm{CISPH}-\overline{\mathrm{H}}$

Fig. 21. (b) ISPH and improved ISPH snapshots of water particles and pressure field during a flip-through impact at $t^{*}=200$ and $t^{*}=500$ 


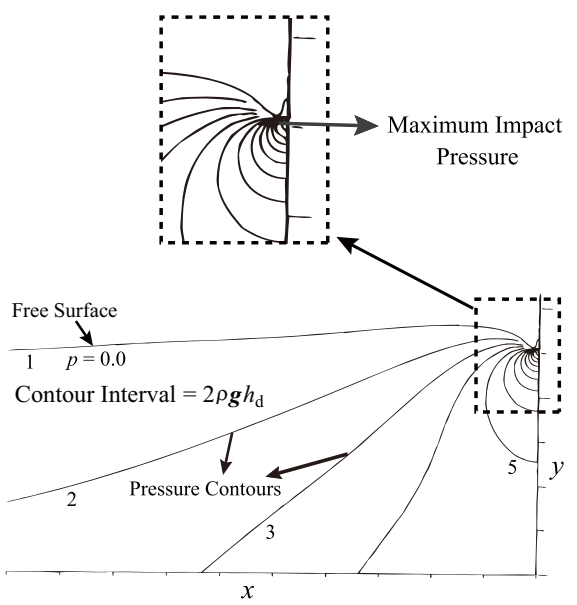

Fig. 22. A schematic sketch of the pressure contours at the time of maximum impact pressure computed by Cooker and Peregrine (1992) 

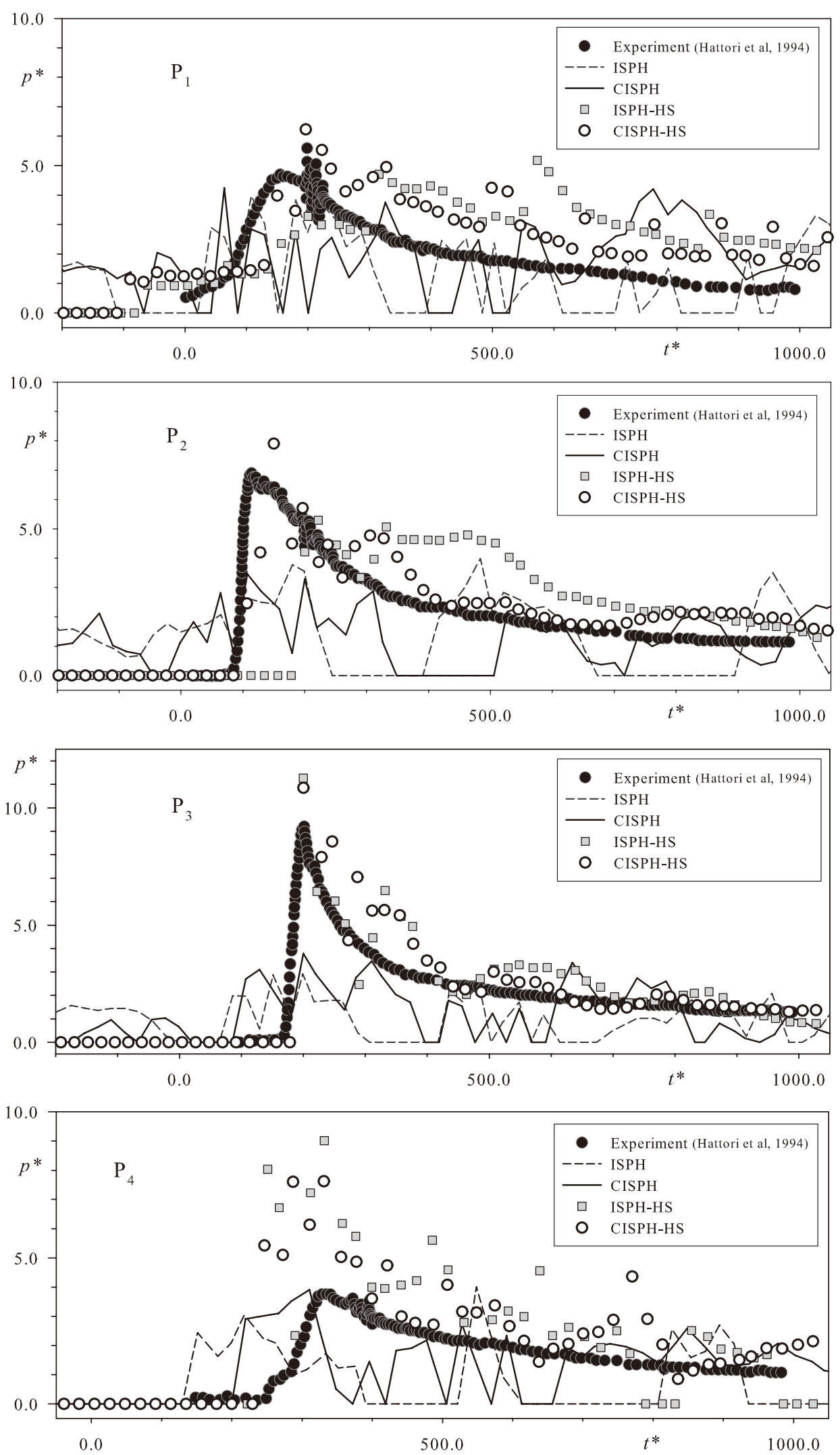

Fig. 23. Dimensionless calculated and experimental wave pressure at different points along a vertical wall during a flip-through impact (Hattori et al., 1994) 